\title{
SUR L'INTRODUCTION
}

D'ESPECES MÉRIDIONALES

1).1.S L..1

\section{FAUNE MALACOLOGIQUE}

DES ENVIRONS DE PARIS

PAll

A. LOGARD et L. GERMAIN

Présenté à l'Académie des Sciences, Belles-Letlres et Arls

lans sa séance du 3 Novembre rgo3.

\section{LYON}

A. REY, IMPRIMEUR DE L'ACADÉMIE

4. RLE GENTIL, 4 

SUR L'INTRODLCTION D'ESPECES NÉRIDIONALES

DANS LA

\section{FAUNE MALACOLOGIQUE}

DES ENVIRONS DE PARIS 



\title{
SUR L'INTRODULTION
}

\author{
D'ESPECES VERIDIONAIES
}

D.NS L.

\section{FAUNE/MALACOLOGIQUE/}

蛋 DES/ENVIRONS/DE PARIS/

\author{
FAR \\ A. LOGARD/ot L. GERMAIN
}

Présenté à l'Académie des Sciences, Belles-Lettres et Arts de Lyon dans sa séance dn 3 Novembre rgo3.

The arsti.

\section{Y ON}

A. REY, IMPRIMEUR DE LACIADEMIE

4. ALE GENTIL, 4

1904 



\title{
SLR L'TSTRODUCTION D'ESPECLS MÉRIDIOXALES
}

DANS LA

\section{FAUNE MALACOLOGIQUE}

\author{
DES ENTIRONS DE PARIS
}

La présence d'espèces malacologiques faisant normalement parlie de la faune méditerıanéenne a déjà été signalée plusieurs fois en divers points de la France, et notamment aux environs de Paris ${ }^{1}$, de Lyon ${ }^{2}$ et d'Anger's ${ }^{3}$. Jamais cependant ces introductions n'ont pris, à notre connaissance du moins, un développement aussi considérable que celui dont nous avons élé lémoins, depuis quelques années, anx environs de Paris. Estimant qu'il serait intéressant pour l'histoire malacologique du Bassin parisien de signaler des faits aussi curieux, nous nous proposons dans ce travail, de relever la liste déjà assez longue de ces espèces, d'étudier' les modifications qu'elles ont pu subir sous l'influence de milieux nouveaux, enfin de rechercher les causes qui ont pu présider à lenr mouvement migraloire.

'Locard (1.), I895. Notices conchyl., XXXV. Une nouvelle station d'espèces méridionales dans le Nord de la France, in L'Echange, XI, p. 121-122,

${ }^{2}$ Locard (A.), is 8 . Nolc sur migrations malacol. env. Lyon, Jyon, gr. in-8, 28 p. - Locard (A.), 1882. Contrib., IV. Sur la présencc espèce méridionale faune malacol. Lyon, gr. in-8, 24 p.

${ }^{3}$ Germain (L.), 1903. Elude Mollusques Maine-el-Loire, Nantes, Introd. VI, p. 36-42. 


\title{
CATALOGUE DES ESPECES
}

\author{
Groupe de l'H. Pisana.
}

\section{Helix Pisana, Müiller.}

IIelix Pisana, Müller, 1774. Verm. IIisl., II, p. 60. -- Locard, 1882. Prodr., 1). 118. - Locard, 1894. Coq. lerr., p. 8o, fig. $y^{3}$.

Une colonie populeuse de l'Ilelix Pisana habite les bords de la Marne, à Charenton. La coquille, bien typique, comme galbe el comme taille (elle atteint 18 millimètres de hauteur pour 23 millimètres de diamètre), présente, dans le test et l'ornementation de très notables différences avec les échantillons de l'Algérie et du Midi. Le test, plus mince, est monochrome, blane brillant, rosé à l'intérieur, orné d'un bourrelet rose bien apparent, absolument semblable à celui des Ilelix Carpiensis, Let. et Bonrs. ${ }^{1}$, et II. Bertini, Bourg. ${ }^{2}$, du Midi de la F'rance. Nous verrons plus loin qu'il 11'en a pas tonjours été ainsi.

L'II. Pisana vil sur les bords de la Marne, le long des talus de la levée, sur un espace assez restreint, s'étendant depuis le pont de Charenton jusqu'aux îles qui encombrent, phis haut, le lit de la rivière. On le rencontre, grimpant sur les liges sèches des arbustes et des hautes herbes. Pendant longtemps nous n'avions pu connaître le mode d'introduclion de ce Mollusque, lorsque M. J. Mabille nous a domné le mot de l'énigme. L'histoire de l'introduction de l'H.

\footnotetext{
1 Letourneux et Bourguignat, 1887. P'odr. malacol. Tunisie, p. 8o et p. 81. - Locard, 1894. Coq. terr., p. 89, - L'II. Carpiensis, qui habite également le Portugal (Loeard), la Tunisie (Letourneux), a étẻ constaté jusqu'en Syrie aux environs de Beyrouth.

"Bourguignal, in Locard, 1882 . Prodr., p. 103 et p. 329. - Locard, เ8,y4. Coq. lerr., 1). s9.
} 
Pisana est, nous dit-il, bien simple : Un de mes amis, vers $\mathbf{1} 668$, avail rapporté d'un voyage dans le Midi un grand panier de ces IIelix dans le seul but de les manger; il lomba malade et sa cuisinière incriminant les malheureux Helix, les jeta sur le talus de la Marne, devant le dépôt des omnibus oì, rencontrant un terrain très propice, ils ont fort bien prospéré ${ }^{1}$. Dans les premiers temps, les individus étaient fasciés comme ceux du Midi, de glande taille et assez souvent déformés. Aujourd'hui, les individus fasciés ont entièrement disparu, quoique la colonie soil très prospère; elle a pu supporter le terrible hiver de $\mathbf{8} 80$ et multiplier beaucoup.

La présence de cette espèce à Charenton est des plus curieuses, l'II. Pisana s'acclimatant très difficilement dans les localités où l'influence maritime ne se fait pas sentir. Elle est trés répandue dans toules les régions méditerranéennes où nous la connaissons en Espagne, en Portugal ${ }^{2}$, en Algérie, en 'Tunisie, en Italie, en Syrie, elc... En France, elle remonte le long des côtes de l'Atlantique (CharenteInférieure, Loire-Inférieure, Côles-du-Nord). On l'a rencontrée accidentellement à Lyon.

\section{Helix Pisanella, Serval.}

IIclix Pisanella, Servain, 1880. Moll. Esp., Porl., p. 1 เ3 (sine descr.). Locard, 1894 . Coq. terr., p. 88.

Cette espèce liabite avec la précédente sur les bords de la

1 Le Dr Jousscaume (Faune malacol, environs de Paris, in Bull. Soc. Zool. France, 1877, p. 217 ) signale le Theba Pisana de la façon suivante: "Cette remarquable espèce a été découverte, il y a quelques jours, à Charenton par M. Kopperhorn, qui nous en a rapporté plusieurs individus de différents âges; ill'a rencontrée sur les bords du canal, à peu de distance de la station du tramway. Il n'est pas douteux que cette espèce, actuellement acclimatéc aux environs de Paris, $y$ ait élé importée depuis très peu d'années. J'ai visité avant 1870 , la localité ou elle se trouve, et je puis certifier qu'elle n'y existait pas à celte ćpoque."

₹ On rencontre aux environs de Cascaès en Portugal, de Tozer en Tuni- 
Marne, à Charenton. Elle y est bien typique el possède le même test blanc jaunâtre monochrome, un peu brillant. L'Ielix Pisanella a élé introduit avec l'H. Pisana, mais il est moins commun.

L'II. Pisanella vil normalement sur lont le littoral méditerranéen; il remonte sur les côtes océaniques françaises, notamment dans le clépartement de la Charente-Inférieure. En dehors de la France, nous lc connaissons en Portugal (Locard), en Espagne (Servain), en Algérie el en Tumisic (Berthier, Letournenx et Bourguignal).

\section{Helix Cuttati, Boungugiat.}

IIelix Cuttati, Bourguignat, in Letourneux et Bourguignat, 1887. I'rodr. Malacol. Tunisie, p. 80 (sine descr.). - Locard, 1894 . Cor. terr., p. 88.

L'Ilelix Cuttati vit à Charenton, sur les hords de la Marne, en compagnie des $M$. I'isana el $I$. Pisanella; il présente le même lest monochrome el le même modıs vivendi que ces deux espèces avec lesquelles il a été introduil.

Une forme minor, ne dépassant pas 15 millimètres de diamètre pour 12 millimètres de hauleur, habite sur le trone des arbres, dans la petile île des Cygnes, à Grenelle, dans Paris même. Celle coquille, qui est évidemment une forme dégénérée, a pu être amenée par les bateaux marchands qui font le service entre le Ilavre el Paris. L'II. Cuttati est, en elfel, une forme du iilloral médilerranéen qui remonte volontiers le long des côles de l'Océan Allantique; nous le comnaissons également du Portugal (Locard), de l'Espagne (Bourguignal) el de la Sicile.

sic el de Beyrouth en Syrie, une variété à larpuelle Bourguignat a donné le nom de var. Thusurosi (Letourneux et Bourguignat, 1887 . Prodr. malacul. Tunisie, 1).81). 
Groupe de l'II. limbala.

Helix limbata, Draparnaud.

Helix limbata, Draparnaud, 1805. Hist. Moll., p. 100, pl.6, fig. 29. - Locard, 189 , . Corg. terr., p. 105, fig, 122-123.

Forme typique, d'un galbe assez constant; outre le type, avec un test assez solide, mince, d'un blane jaunâtre, orné d'une bande blanche sur la carène, on rencontre fréquemment la var. Sarratina, Moq.-Tand., de couleur fauve, plus oll moins foncé.

'Très répandı dans le bois de Clamarl, le long dı mur dı parc aérostatique de Mendon, entre la fontaine Sainte-Marie et l'étangn de Trivaux; presque exclusivement sous les feuilles de Rubns. Celle espece, qui a été introduite vers 1872 par M. Arthon, s'est très rapidement développée dans toute cette région oì elle semble définitivement acclimatée.

Cette forme d'origine méridionale se retrouve aujourd'hui dans presque toute la France littorale et centrale, depuis la région pyréréenne jusque dans le Calvados oì elle aurait élé introduite par de l'Iopital; on l'a signalée dans le Maine-etLoire, la Sarthe, l'Allier, la Mayenne, les Deux-Sèvres, la Tienne et, plus an sud, dans l'Ariège, le Gers et la HauteGaronne.

$$
\text { Groupe de l'll. Terveri. }
$$

Helix Augustiniana, Bourguigrat.

Ilelix Augustiniana, Bourguignat, in Servain, 1880 . Yoll. Esp. Port., p. 73 . - Locard, 189 ;. Coq. terr., P. 208, fig. 26y-270.

Le type de cette espèce a étú découvert en Algérie, près de Bône, sur les ruines de l'ancienne Hippone. Le seul échantillon de la plaine Saint-Denis que nous possédions est bien typique; il porte, sur le milieu du dernier tour, une 
très étroile bande à peine colorée, peu distincte. Nous avons observé cette même var. zonata chez des échantillons de Menton (Alpes-Maritines) et de Saint-Affrique (Aveyron).

En France, nous connaissons l'Helix Augusliniana dans les départements des Alpes-Marilimes, du Var, des Bouchesdu-Rhône, du Gard et de l'Aveyron, oiı il vit en colonies assez populeuses, mais localisées; il remonte, assez rarement, le long de l'Allantique el de la Manche. M. le Dr Servain a signalé, aux environs de Badajoz, en Espagne, une forme minor de cette espèce qui. habite également les environs de Bône, en Algérie (IVesterlund).

\section{Helix limbifera, Locard.}

Helix limbifera, Locard, 1894. Coq. terr., p. 209.

L'Ilelix limbifera possède, autour de Paris, un galbe un peu plus élevé et une taille un peu plus petite que le type du Midi de la France. Le lest est assez brillant, blanchâtre ou jaunacé, un pen épais, crétacé, très généralement orné d'une seule bande carénale brune, brillante, étroile, avec quelquefois deux ou trois bandes infra-carénales peu apparentes. On rencontre également à Gentilly, ume var. unicolor. Diam. : r 4 -17 millim, ; haut. : 9-12 millim.

Çà et là, sur les talus cxposés au Midi; grimpe sur le Cenlaurea calcitrapa, Lin. et le Carduus tenuiflorus, Lin.: champs près le fort de Bicêtre; sur les luzernes, dans les champs, près le fort de Montrouge; talus à Gentilly; talus des fortifications, porte de Patay; bouds de la Marne, entre le confluent el Charenton. Dans cette dernière localité, les échantillons sont plus typiques et ornés de bandes subtransparentes assez larges, parfois partiellement soudées; on y rencontre également une var. minor ne mesurant que 13 I / millimètres de diamètre pour 9 millimètres de hauteur. 
Nous connaissons l'H. limbifera dans les départements des Alpes-Maritimes, du Var, des Bouches-du-Rhône, de Vaucluse, des Pyrénées-Orientales, des Basses-Pyrénées el de la Gironde. D'après la collection Bourguignat, celle espèce remonte le long des côtes océaniques de France. Elle se retrouve aussi en Portugal (Locard).

\section{Helix terraria, LocARD.}

Ilelix terraria, Locard, 1894 . Coq. terr., p. 209.

Nous possédons deux échantillons de cette espèce, recneillis sur les hzernes, dans un champ, près le fort de Montronge. Ils sont de petite taille, assez typiques, ì spire très surbaissée; le test est blanchàtre; l'un des individus possède six bandes brunes : une bande supra-carénale, lrès large, continuée en dessus et cinq infra-carénales étroites; l'autre coquille n'a qu'une bande supra-carénale étroite, continnée en dessus. Diam. : 1/15 1/2 millim.; haut. : 9 1/4-9 $1 / 2$ millim.

Nous avons recueilli récemment dans les carrières d'Arcneil, près l'Aqueduc, de magnifiques échantillons de cette espèce, mesurant 17 millimètres de diamètre pour 12 millimètres de hauteur, ornés de trois larges fascies brunes subtransparentes: une fascie supra-carénale continue en dessus et deux infra-carénales, plus développées, presque sondées aux environs de l'ouverture.

L'Ilelix terraria nous est connu dans les départements des Alpes-Maritimes, diu Var, des Bouches-du-Rhoone, de Vaucluse, du Gard et de l'Ilérault; il habite également le Porlugal (Locard).

Helix leviculina, Locand.

IIelix leviculina, Locard, 1894 . Coq. terr., p. 210.

Le type de cette espèce, si reconnaissable ì son galbe 
très déprimé, légèrement lectiforme, presque plan en dessus, habite sur les plantes sèches des bords de la mer, aux Catalans, à Marseille. La coquille des rives de la Marne, à Charenton, est une forme minor mesurant de 9 丸̀ 12 millimètres de diamètre pour 6 1/2 a 7 I/2 millimètres de hauteur. Elle est bien typique et présente un test blanc erétacé, un peu épais, orné de deux ì cinq bandes brunes élroites, la première, parfois continuée en dessus'. Nous connaissons cette forme minor, de Nice, d'Orgon, d'Arles.

Nous arons également recueilli à Arcueil, près de l'Aqueduc, un échantillon mesurant is millimètres de diamètre pour 10 millinitres et demi de hauteur, au test blanc, finement strié, orné d'une bande marron clair, légèrement subcarénale et de deux bandes infra-carénales, très élroites, presque obsolètes aux environs de l'ouverture, qui se rapproche beaucoup plus du type de la station des Catalans, à Marseille.

Le type de l'Lelix leviculina n'a encore été signalé que dans les Alpes-Maritimes, le Tar, les Bonches-du-Khône el le Lot-ct-Garonne.

\section{Groupe de l'II. Insiana.}

Helix Jusiana, Bolngulgrat.

Helix Jusiana, Bourguignat, ap. Locard, 1885. In Bull. Soc. Malacol., II, p. 7 fi. - Locard, 1894 . Coq. terr.. p. 210, fig. 27 1-272.

Celle espèce, si reconnaissable à son gallye globuleux, un peu conique en dessus ct à son test blanc brillant porcelanisé, est très rare aux environs de Paris. Le seul échantillon que nous possédous est bien typique; son test blanc un peu brillant, est très finement striolé, légèrement jamâtre en

'Chez la coquille du Midi, le nombre des bandes ornementales varie entre 6 el 8 . 
dessous el rosé aux environs de l'ouverture qui est garnie d'un fort bourrelet fauve, roux clair. Diam. : so millim.; hant. : 33 millim.

Buissons de clôture, en haut du talus du chemin de fer de Ceinture, rue Regnault à Paris même.

Nous connaissons cette eqquille des départements du Var, des Bouches-du-lihône, de Vancluse, du Gard et de l'Hérault. Elle est également acelimatée ì Lyon. M. Westerlund l'a retronvée, en Algérie, à l'étal fossile.

\section{Helix Salentina, I. BuANC.}

Melix Salentina, 11. Blane, in Locard, r885. In Bull. Soc. Malacol., I1, 1.-73. - Locard, 389 . Cor. terr., p. 21 .

Nous en avons recueilli de magnifiques éehantillons parfaitement adultes, à Choisy-le-Roi, rue de la Pépinière. Ces coquilles sont bien typiques, de laille petite ou moyenne; leur test est blanc jaunátre brillant, agrénenté, sur le dernier lour, de zones fiunes phus transparentes que le reste de la coquille, parallèles aux stries qui sont fines el assez régulières; l'ouverture présente un bourrelet interne brun-roux assez développé el bien coloré. Diam. : $15-18$ millim.; haut. : 1 2-14 millim. Comme dans les éeliantillons de Lyon ${ }^{1}$, nous avons observé fréquemment un double bourrelet interne.

Vit sous les tonffes d'orties, en compagnie de l'IIelix ericetorum, Müll., rare: Choisy-le-Roi, rue de la Pépinière, passage à niveau du clıemin de fer du P.-L.-M., sur la route de Créleil; Arcueil, chemin près de l'aqueduc, en allant ver's les carrières.

L'Il. Salentina est une forme méridionale l'épandue en Italie, en Sicile, en Espagne, en Grèce el en Algérie. En

1 Locard (A.), i885. Matér. hist. Malacol., IV, in Bull. Soc. Malacol. France, II, p. $; 6$. 
France, nous la connaissons des départements des AlpesMaritimes, des Bouches-du-Rhône, de Vaucluse, du Gard, de la Drôme, de la Corrèze, du Lot-et-Garonne et de la Vendée.

En 1840 , elle avait été observée par Terver à Lyon, dans la presqu'île de Perrache et aux litroits; il la désignail sous le nom d'H. variabilis; elle semblail disparue, mais à partir de 1870 , elle s'est acclimatée définitivement dans un autre quartier, sur les lalus dı comrs Lafayette an voisinage du chemin de fer.

\section{Helix calculina, Locard.}

Helix calculina, Locard, 1894. Coq. torr., p. 211.

Le seul échantillon, de Gentilly, que nous possédons est bien typique; son test est blane jaunâtre, un peu brillant, surtout en dessous, orné d'une vague bande supra-carénale blanchâtre, à peine sensible. Diam. : 1/4 millim.; haut.: 13 millim.

L'Helix calculina est une espèce peu commune, que l'on reneontre surtout dans les départements méridionaux (Alpes-Maritimes, Var, Bouches-du-Phône, Vaucluse, Gard, Lol-et-Garonne), mais qui remonte un peu le long des côtes de l'Atlanlique (Charente-Inférieure, Vendée).

Helix acomptia, Bourgugnat.

Ilelix acomplia, Bourguignat, r864. Malacol. Algérie, I, p. 218, pl. 24 , fig. 17-21. - Locard, 1894. Coq. terr., p. 212.

Cette forme, si particulièrement globuleuse-conique, au test porcelanisé, a été signalée ponr la première fois en Algérie par le regretté Bourguignat. Nos échantillons, trouvés à Lagny-Thorigny, sur les talus du chemin de fer, à 
environ 300 mètres à l'ouest de la gare, sont absolument conformes à d'autres individus de l'llérault el de la Charente-Inférieure; ils sont plus petits que ceux d'Algẻrie et ne dépassent pas 15 millim. de diamètre: sur l'un d'eux on distingue en dessous des traces apparentes de trois bandes roux-clair, les deux plus inférieures lrès élroites et coutimues, la supéricure plus large el plus confuse, s'atténuant complètement à partir du milieu du dernier tour. Nous désignerons celte variation de coloration sous le nom de var. zonula. Cette variation n'est pas très rare chez l'Helix acomptia : c'est me des formes d'Ilelix ì test poreelanisé qui ont le plus de tendance à avoir des bandes colorées visibles seulement en dessous du dernier tour de la coquille; nous l'avons observée sur des échantillons provenant d'Hyères, Palavas, Saint-Martin-rle-Ré, Cabourg, mais uniquement chez la var. minor.

Cette espèce algérienne a été retrouvée en France dans plusieurs stations fort dispersees, oì elle constitue des colonies peu populeuses : nous la connaissons dans les départements de Vaucluse, Aude, Iéraul, Haute-Garonne, Areyron, Lozère, Charente, Charente-Inférieure, Calvados, etc.

Helix acomptiella, Locard.

Helix acomptiella, Locard, 1894 . Coq. terr., p. 2 i2, fig. $275-2-6$.

Cette espèce vil, aux environs de Paris, en colonies assez dispersées, médiocremeut populeuses. Les échantillons, bien typiques, sont parfaitement conformes à la figuration citée dans notre synonymie. Comparés aux lypes algériens, ils présentent un test moins blane, moins brillant, beaucoup moins épais et crélacé. l'Ilelix acomptiella atteint parfois, notamment à Charenton, une forte taille, son galbe restant 
d'ailleurs parfaitement typique, bien conique en dessus. Diam. : 13-16 I/2 millim.; haut. : I2-1 / millim. Nous avons recueilli, sur les Luzernes, près le fort de Montrouge, une variété minor presque un tiers plus petite (Diam. : 10-11 millim.; haut. : 8-10 millim.)

Pen commun; de préférence sur les Ombellifères : Paris; Square Alboni ì Passy; Lalus des fortifications près la gare d'Orléans: champs et talus à Gentilly; Montrouge; Choisyle-Roi, près du passage á niveau du P.-L.-M.; bords de la Marne a Charenton; la plaine Saint-Denis; Arcueil, carrières, près de l'aqueduc.

L'H. acompliella est une forme ordinairement assez rare, mais qui remonte le long des côtes océaniques françaises. Elle nous est comnue des départements de l'Aveyron, du Tarn-et-Garonne, de la Corrèze, de la Gironde, de la Charente-Inférieure et du Finistère. Elle habite également l'Algérie (W'esterlund) et le Portugal (Locard).

\section{Helix suberis, Bourguignat.}

IIelix suberis, Bourguignat, in Locard, 1885. Bull. Soc. Malacul., II, p. 54. - Locard, 1894. Coq. terr., p. 213.

L'Ifelix suberis est une forme bien constante, rare aux environs de Paris, où les échantillons parfaitement typiques, d'un galbe relativement peu globuleux, subdéprimé, présentent comme dans le Midi "cette indieation d'une fausse earène à peine sensible sur le profil du dernier tour, mais rendue apparente au regard par la présence d'une ligue blanchâtre un peu brillante qui règne sur ce point'. " Le test est roux sale, finement costulé; l'ouverture est garnie d'un bourrelet rosé. Diam. : 16-18 millim.; haut. : 10-13 I/2 millim.

${ }^{1}$ Locard( $\left.\Lambda.\right), 1885$, Matér. hist. Malacol. fr., in Bull. Soc. Malacol, France, II, p. 56. 
Rare. Talus des fortifications : porte de Vitry; porte de Palay.

Celte espèce, peu répandıc, n'a encore élé signalée qu'aux environs d'Hyères (Var) et de Nîmes (Gard). Eille s'est également naturalisée à Sainte-Catherine-le-Fierbois, dans le département de I'Indre-ct-Loire.

\section{Helix Evenosi, Bourgulgnat.}

Melix Evenosi, Bomrguignat, ap. Locard, a 885. In Bull. Soc. Malacol., Il, p. 56. - Locard, 1894 . Cor. terr., p. $213, \mathrm{fig} .277,278$.

Coquille d'un galbe voisin dı lype méridional, mais à dernier lour moins globuleux; le test est solide, un peu crétacé, blanc brillant, finement costulé, parfois légèrement malléé sur le dernier tour. L’ombilic très étroil, est plus recouvert par suite d'un épaississement plus accentué du bord colımellaire; la coquille passe ainsi à la var. subumbilicata locard ${ }^{1}$, variété que nous connaissions déjà de Graveson (Bouclies-du-Rhône) et de Port-Sainte-Marie (Lot-elGaroune). Diam. : 8 millim.; hant. : 12 millim.

Très rare, ¿̀ Argentenil el sur les Luzernes aux environs du fort de Montronge oi cette forme vit en eompagnie des Ilelix ademata, II. terraria el II. limbifera.

Nous connaissons l'H. Evenosi dans le Var, les Bonchesdı-khône, Vaucluse, le Gard, l'IJérault, la Haute-Garonne, le Lot-el-Garonne, la Charente-Inférieure el, en dehors de la France, en Porlugal (Locard).

Helix ademata, Bourguigrat.

Ilelix ademala, Bourguignal, ap. Locard, ı885. In Bull. Soc. Malacol., II, [.65. - Locard, 1894 . Cog. Ierr., p. 214.

Celle espèce, du groupe espagnol de l'Helix Castroiani,

1 Locard (A.), เ885. Matér. Malacol. française, in Bull. Soc. Malacol. France, II, p. 58. 
Servain, est abondante à Choisy-le-Roi, près le passage à niveau du chemin de fer P.-L.-M. Nos échantillons présentent un assez grand polymorphisme portant sur le galbe qui est plus ou moins globuleux el surtout sur la spire qui est plus ou moins haute tout en restant cependant, chez la grande majorité des individus, notablement plus basse que dans les colonies méridionales. Le tesl est d'un beau blanc brillant, parfois un peu jaunâtre, crélacé, solide, quoique nolablement plus mince que chez les coquilles du Midi. La taille reste petite. Diam. : 14-16 millim.; haut. : 10-12 mil.

L'H. ademata vit en très grande abondance à Choisy-leRoi, près le passage à niveau du chemin de fer de P.-L.-M., sur les chardons, et l'on peut voir parfois des espaces de plusieurs mèlres carrés presque disparaître sous une couche d'un bean blane formée d'échantillons de celle espèce. On la rencontre encore, mais beaucoup moins commune, sur les Luzerues près le fort de Montrouge et dans la plaine Saint-Denis.

Nous connaissons cette même forme des Alpes-Marilimes, du Var, des Bouches-du-lihône, du Gard, de la HauteGaronne el de l'lle-de-Ré. Bourguignat l'a également signalée en Algérie, à Oran.

Helix Kalona, Bentuer.

Melix Ëalona, Berthier, in Locard, 1894. Coq. terr., p. 214.

Cette forme provençale est assez bien caractérisée autour de Paris, mais présente un galbe plus globuleux quoique bien constant, ne permettant de constater que de légères variations dans l'allure de la spire qui est plus ou moins conique. Le test est un peu mince, subtransparent, blane, subcrélacé, assez brillant, un peu roux clair en dessous. Diam. : 12-15 millim.; haut. : 9-10 millim. 
Rare: talus ombragés des fortifications entre la porte de Patay et le chemin de fer d'Orléans; Areueil, chemin se dirigeant vers les carrières, près de l'Aqueduc.

L'H. Kalona habite normalement les Bonches-du-Rhônc, Vaucluse, le Gard el l'Hérault.

\section{Helix limarella, Hagenmïlter.}

IIelix limara, var. limarella, Hagenmüller, in WesterIund, 1889. Fauna palaar., I, p. 178 .

- limarella, Locard, 18y'. Coq. terr., p. 215.

Coquille de taille moyenne, généralement plus globuleuse que celle des Bouches du Rhône, à lest notablement moins solide; le bourrelet interne, peu coloré, n'est que faiblement indiqué. Diam. : 13-14 1/2 millim.; laut. : 9-1 1 millim. L'aspect déprimé de la spire provient surtout des trois premiers tours et du sommet qui sont comme écrasés. Nous possédons une var. major de Choisy-le-Roi, de même galbe, mais mesurant 18 millim. de diamètre maximum.

Rare: vit sur le gazon des Lalus exposés au Midi : à Gentilly, à Choisy-le-Roi (route de Créteil) el à Paris (Portes de Vitry et de Patay.)

Nous connaissons cette forme dans les départements des Alpes-Maritimes, du Var, des Bouches-du-Rhòne, de Vaucluse, du Gard et de la Haute-Garomne.

\section{Helix subtassyana, Locand.}

IIclix Tassyana, Fagot, in Locard, 1885. Bull. Soe. Malacol., II, p. 70 (non Bourg.). - IIelix sublassyana, Locard, i 894. Coq. lerr., p. 215.

Cetle espèce, découverte au mont Alarie dans le départoment de l'Aude, par M. Paul Fagot, est rare dans la région parisienne. Les échantillons que nous avons récoltés ¿Arcueil, près de l'Aqueduc, mesurent 13-14 millim. de 
diamètre pour 10 millim. de hauteur; ils sont done de taille normale; leur galbe est assez nettement déprimé, leur test est blanc, légérement brillanl, sans traces de fascies, orné de stries longitudinales ondulées, très fines et très irrégulières.

L'Ilelix sublassyana est me forme rare, habitant les départements de l'Aude el de Vaucluse.

\section{Helix Mendranopsis, LoCaRD.}

IIelix Mendranopsis, Locard, 1894. Coq. lerr., p. 215.

L'Ilelix Mendranopsis est une des formes méridionales les plus répandues autonr de Paris oin elle vit en colonies populeuses dans un grand nombre de localilés, en compagnie de l'Il. Cyzicensis, Gall. La coquille parisienne présente ce galbe un peu déprimé, quoique subglobulenxconique daus son ensemble qui caractérise si bien celle espèce. Le test est blauc porcelanisé. un pen jaunàtre en dessons, brillant, assez épais. Diam. : 13 1/2-17 millim,; haut. : 11-r/4 millim. On observe un polynorphisme portant sur le galbe qui est plus on moins élevé, sur l'allure de la spire, ele... mais surtoul sur la taille qui permet de dislinguer une var. major alteignant 20 millim. de diamètre pour 16 millim. de hauteur (Choisy-le-Roi, près le passage à niveau du P-L-NI; Sennevières), et une var. minor un tiers plus petite: diam. : 12-13 millim. : hant. : 9 1/2-10 millim. (Boulevard Victor, à Paris; talus à Gentilly; Choisyle-Roi, passage ì nivean du P-L-M.)

Vit sur les talus exposés an soleil, parmi le gazon, sur les ombellifères, les chardons et les Centaurea calcitrapa, L. Très comnun ; souvent en colonies extrêmement populeuses : Paris, talus des fortifications; boulevard Victor, porte 
de Gentilly, porte de Patay, porte d'Austerlitz, près la gare d'Orléans, porte d'Arcueil ; Square Alboni, à Passy; Champs près le fort de Montrouge; près le fort de Bicêtre; talus à Gentilly; Choisy-le-Roi, route de Créteil, près le passage à niveau du P-L-M; bords de la Marne à Charenton; Arcueil ; Asnières ; Courbevoie; Argenlenil (Seine-et-Oise); Sennevières, près Nantenil-le-Haudouin (Oise); Lagny (Seineet-Marne.)

L'II. Mendranopsis commun dans preque tont le Midi (Alpes-Maritimes, Var, Bouches-du-Rhône, Gard, Hérault, Pyrénées-Orientales, Haute-Garoune, Lot-et-Garonne), remonte sur les côtes de l'Océan Atlanlique et même de la Manche (Gironde, Charente-Inférieure, Ile de Ré, BelleIsle, Vendée, Indre-et-Loire, Calvados, îles Chausey) ; on le trouve aussi accidentellement à Lyon.

Helix Nemausensis, Bourguigrat.

IIelix Nemausensis, Bourguignat, in, Locard, Isgł. Cart. Lerr., p. 216. fig. $279^{-2} 80$.

Comme l'espèce précédente, l'II. Nemansensis est une forme très répandue autour de Paris. Elle y présente un galbe notablement plus élevé qu'en Provence; le test est blanc, porcelanisé, solide, assez épais, brillant et très finement striolé; l'ouverture est garnie d'un bourrelet un peu saillant, rougeàtre, brillant. Diam. : ı3-ı 5 millim.; haut. : I0- 12 millim.

Nous distinguerons chez cette espèce : une var. depressa caractérisée par un galbe très déprimé et une ouverture relativement plus grande (talus des fortifications, boulevard Victor, à Paris); une var. major très netle : diam. : I7 millim.; haut. : 12 1/2 millim. (talus à Gentilly.) 
Très commun, sur les talus exposés au soleil : Paris, talus des fortifications, boulevard Victor, porte de Vitry, porte de Gentilly, rue Regnault, talus du chemin de fer de Ceinture; Square Alboni, à Passy; champs près le fort de Montrouge; près le fort d'Ivry; près le fort de Bicêtre; talus à Gentilly; Choisy, route de Créteil près le passage à niveau du P.-L.-M; bords de la Marne à Charenton; Areueil, chemin des carrières, ete.

L'II. Nemausensis nous est connu des départements du Var, des Bouches-du-Rhône, du Gard, de Vaucluse, de l'Hérault, du Lol-el-Garonne, de la Charente-Inférieure, de la Vendée et du Finistère.

Groupe de l'II. Avenionensis.

\section{Helix Avenionensis, Bourguignat.}

Ilelix Avenionensis, Bourguignat, ap. Locard, 1885. In Bull. Soc.Malacol., II, p. 65. - Locard, 1894 . Coq. terr., p. 216 , fig. $281-282$.

Cette jolie petite forme, distinguée pour la première fois par Bourguignat, s'est largement répandue aux environs de Paris, particulièrement sur les talus des forlifications. Elle a conservé son galbe bien typique, mais le dernier tour est relativement moins développé el moins globuleux. Le test est blane, assez brillant, peu épais. Diam. : 12-14 I/2 millim. ; haut. : 10 1/2-11 1/4 millim. Une var. minor de même galbe, à test plus mince et un tiers plus petite (Diam. : 9 1/2-10 1/2 millim.; haut. : 8-9 millim.), vit sur les planches de clôture des terrains vagues, rue Barrault à Paris.

Très commun sur les gazons des talus, etc. : Paris, talus des fortifications, près la gare d'Orléans, porte de Vitry, porte de Gentilly, rue Barrault; square Alboni, à Passy; 
Gentilly, Areueil; Montrouge; bords de la Marne à Charenton; Choisy-le-roi, route de Créteil près le passage à niveau du P.-L.--I.; le Vésinet.

L'llelix Avenionensis est une des formes de Variabiliana qui remontent le plus haut sur les côtes de la Manche. Nous connaissons en effet cette espèce de Boulogne dans le Pasde-Calais. Elle vit encore dans les départements des Bouches-du-Rhône, du Var, de Vaucluse, du Gard, de l'A veyron et remonte, le long des côtes océaniques, dans la CharenteInférieure, la Loire-Inférieure et la Vendẻe.

\section{Helix Guideloni, Bovrguignat.}

IIelix Guideloni, Bourguignat, in Locard, 189 . Coq. terr., p. 217.

Cette espèce, d'un galbe un peu haut, et dont le dernier tour est raguement subcaréné ì sa naissance, est bien eonforme au type méridional; cependant son test est moins brillant; il est blane, très légèrement porcelanisé, rarement jaunâtre. Quelques rares échantillons sont ornés d'une vague bande supra-carénale brune, réduite à des points. Nous désignerons cette variété sous le nom de zonata. Diam.: 10-12 millim.; hauteur : 7 1/2-10 1/2 millim.

Commun : Paris, talus des fortifieations, près la gare d'Orléans, porte de Vitry, porte de Gentilly; Square Alboni, à Passy; Choisy-le-roi, près le passage à niveau du P.-L.-M. ; champs près le fort de Montrouge; talus à Gentilly.

Nous connaissons l'Helix Guideloni dans les départements suivants : Bouches-du-Rhône, Hérault, Gers, HanteGaronne, Lot-et-Garonne el Loire-Inférieure. Il se montre accidentellement à Lyon et se retronve dans le Portugal (Locard). 
Helix ambielina, de Cuhrpentier.

IIclix ambielina, de Charpenties, in Paladilhe, 1867. Miscell. Malacol, 1). 41 (sine descrip.). - Locard, 1894. Cor. terr., p. 217.

Le seul échantillon que nous possédions de cetle espèce a été recueilli vivant et bien adulte sur le talus des fortifications, non loin de la gare d'Orléans. Il est parfaitement conforme, comme galbe et comme taille, au type méridional '; son test est notablement moins brillant, son ouverture est garnie d'un assez fort bourrelet rosé. Diam. : 13 millim.; haut. : 10 millim.

L'H. ambielina n'a pas encore été signalé sur les côtes de l'Allantique; nous ne le connaissons que sur le littoral méditerranéen, dans les départements des Alpes-Maritimes, des Bouches-du-Rhône, de l'Aude et de l'Hérault où il est assez rare.

Helix fera, Letourveux el Bourguigrat.

IJelix rera, Letourneux et Bourguignat, 1887. Prodr. Malacol. Tunisie, p. 5o. - Locard, 1894 . Cor. terr., p. 217 , fig. $283-284$.

L'Helix fera, découvert d'abol'd en Tunisie aux environs de Tebourba (Berthier) et de Carthage (IIagenmüller), est une petite forme déprimée, convere-tectiforme en dessus, surtout caracteriséc par son dernier tour bien anguleux et comprimé sur les trois quarts de sa longueur. Cette espèce,

1 Nous prenons pour type les échantillons adressés par de Charpentier lui-même à Bourguignat; ces ćchantillons sont aujourd'hui au musée de Genève; ce sont eux qui ont servi de terme de comparaison à Paladilhe. Quant aux échantilions de la collection de Charpentier au musée de Lausanne, ils sont, nous éerit M. le marquis de Monterosato, tout différents; mais la collection du savant malacologiste suisse avant d'être installée comme elle l'est aujourd'hui, a subi de telles viscissitudes, que nous croyons prudent de nous en tenir au type de la collection Bourguignat. 
bien typique dans nos environs, y présente un lest blanc porcelanisé, assez Jrillant. Diam.: 9-13 millim.; haut.: 6-8 $1 / 2$ millim. Nous signalerons une var. minor ne mesurant que $8-8 \quad 1 / 2$ millim. de diamètre pour 6 $6 \mathrm{I} / 2$ millim. de hauleur, mais néanmoins pourvue d'un fort bourrelet interne. (Planches de clôtures, rue Barrault ì Paris; Lalus à Gentilly.)

L'II. fera est une forme très robuste qui s'est rapidement acclimatée autour de Paris: c'est aujourd'hui l'espèce de ce groupe la plus répandne. Elle vit en colonies très popıleuses sur les tiges siches des grandes ombellifères el des chardons, plus rarement sur le gazon ou les orties. Nous l'avons recucillic abondamment dans les localités suivantes: Paris, talus des fortifications, près Orléans-Ceinture, porte de Vitry, porte de Gentilly, porte d'Auteuil, rue Barrault, square Alboni, à Passy, elc...; champs à Montrouge; ̀̀ Gentilly; à Choisy-le-Roi, route de Créteil; à Châtillon; bords de la Marne à Charenton, etc...

Nous commaissons l' $/$. fera des départements des AlpesMarilimes, des Bouches-du-Rliône, du Vaucluse, du Gard, du Lot-et-Garonne, de la Vendée, de l'Ille-el-Vilaine, de la Seine-Inférieure et des îles Chausey dans la Manche. Il remonte accidentellement jusqu'à Lyon.

\section{Helix Granzonensis, Boungulgnat.}

Ilclix Grannonensis, Bourguignat, in Servain, 1880, Moll. Esp., p. 104. Locard, $18 y$. Coq. terr., p. 218 , fig. $28 j-286$.

Cette forme beaucoup plus rare que la précédente reste bien lypique; ce n'est que par exception que l'on remarque, clıez quelques individus, un dermier tour très vaguement subcaréné aux environs immédiats de l'ouverture. 'Test blane, quelquefois jaunacé, assez brillant, relativement minee. Dian. : 11-13 millim, ; haut. : 9-1 1 millim. 
Rare, en colonies peu populeuses: Paris, talus des fortifications, boulevard Victor, porte de Vitry; square Alboni, à Passy; talus à Gentilly.

L'Helix Grannonensis est une forme méridionale assez commune, qui remonte volontiers le long des côtes de l'Atlantique. Nous en avons observé la présence dans les départements des Alpes-Maritimes, dı Var, des Bouchesdu-Rhône, de Vauchse, dı Gard, des Pyrénées-Orientales, de la Haute-Garonne, de la Dordogne, de la Charente-Inférieure, de la Vendée, de la Loire-Inférieure, du Finislère et du Calvados. Cette même forme a été également retrouvée en Espagne, en Portugal, en Italie (Locard), en Algérie et en Tunisie, sur le littoral, près de Gabès (Letourneux).

Groupe de l'Il. variabilis.

Helix variabilis, Draparnaud.

IIelix variabilis, Draparnaud, 1801. Tabl. Moll., p.73.-Draparnaud, 1805. IIist..1oll., p. 84, pl. 5, fig. 11-12. - Locard, 1894. Coq.terr., p. 218, fig. $287-288$.

On a confondu sous ce nom un grand nombre de formes absolument différentes, mais nous prendrons pour type la coquille figurée par Draparnaud ${ }^{1}$. A Sennevières, les échantillons sont d'un galbe très nettement caractérisé, mesurant de 16 à 18 millim. de diamètre pour 13 à 14 millim. de liauteur; les bandes sont d'un brun clair. Les coquilles de Durtal, en Maine-et-Loire, ont 17 millim. de diamètre et 13 millim. de hauteur; elles constituent done une forme un peu plus surbaissée que le type, correspondant à une variété à laquelle l'un de nous a donné le nom de Durtalensis².

1 Locard (1.), 1895. Ipsa Draparnaudi Conchylia, p. 108.

${ }^{2}$ Germain (L.), igo3. Etude Moll. Maine-et-Loire, p. 124. 
Nous connaissons cetle même variété aux environs de La Rochelle ${ }^{1}$.

L'Helix variabilis est une forme essentiellement méridionale (Alpes-Maritimes, Var, Bouches-du-Rhône, Vaucluse, Hérault, Haute-Garonne, Lot-et-Garonne, etc...), que l'on retrouve en Portugal (Locard), en Algérie et en Tunisie [environs de Tunis (Bourguignat), Menzel-Tenen (Letoulneux), etc... el qui remonte sur le littoral de l'Océan Allantique au moins jusqu'ì Cherbourg (Gironde, Charente-Inférieure, Maine-et-Loire, Yendẻe, Manche, Calvados).

\section{Helix lutosinula, LocARd.}

Ilelix luteata, Locard, 1894. Coq. terr., p. 219. (Non Parreys, in: Pfeiffer. 1857. Malack. Blätter, IV, p. 87).

- lutosinula, Locard, rgo3. IIss.

Cette magnifique espèce, au galbe subglobuleux, un peu déprimé, et dontles tours, très peu convexes, presque plans en dessus, sont séparés par des sutures peu prolondes, est très rare aux environs de Paris. Le seul échantillon que nous possédons vivail sur un talus bien exposé an midi, non loin de l'avenue Victor-Hugo, à Gentilly. Il est bien typi-

1 La présence de eette espèce a été déjà indiquée par M. le Dr Jousseaume (Jousseaume, 1878. Faune malacologique des environs de Paris, huitiène article, (Bull. Soc.Zool. France, p. 210) sous le nom de Thebavirgata: "Il est très abondant aux environs de Paris, et sa découverte en est facile, car il habite surtout les endroils secs et découverts. On le trouve souvent groupé le long des plantes ou des barreaux de clôture, ru'il recourre quelquefois complètement. Il n'est pas rare de reneontrer des plantes sur les branches desquelles ils s'amassent en si zrand nombre quils forment des grappes dont le volume atteint somvent la grosseur d'un cuf. Il suffit de visiter les bords du canal à Saint-Denis ou les talus du chemin de fer ì Grenelle pour se le procurer. La présence du Theba virgala aux environs de Paris, où il est encore cantonné dans celtaines localités, n'a été constatée que depuis (juelques années; i] a dù être imporlé depuis Ia création des chemins de fer, qui ont permis d'apporter des fourrages des localités oủ il se trouve. » 
que comme galbe et comme taille (diam. : 18 millim.; haut. : 15 millim.). Son test, assez solide, blanchâtre en dessus, jaunacé en dessous, est brillant, orné d'une large fascie subcarénale brune el de cinq fascies infracarénales, plus étroites, également brunes. Toutes les bandes qui ornent le test de cette coquille s'élargissent aux environs de l'ouverture; la fascie supracarénale seule est continuée en dessus.

L'Helix lutosinula est une forme rare, que nous ne connaissons encore que dans les Alpes-Maritimes, le Var, les Bouches-du-Rhône, Vaucluse, le Gard et à l'île de Ró.

$$
\text { Groupe de l'Il. Xalonica. }
$$

Helix Xalonica, Servar.

IIelix Xalonica, Servain, 1880. Moll. Esp. Port., p. 102. - Locard, 189\%. Coq. terr., p. 222, fig. 293-294.

Belle espèce, assez rare autour de Paris; de galbe et de taille normaux, les échantillons présentent un lest mince, beaucoup moins chaudement coloré que dans le Midi; on observe une diminution très sensible dans la largeur des bandes brunes qui ornent le tesl; ces bandes ont évidemment tendance à disparaitre. Diam. : 12-16 millim.; haut. : 9- 2 millim.

Assez rare; en colonies peu populeuses sur les Composées ou les Ombellifères: Paris, talus des fortifications, porte de Vitry, porte de Gentilly; square Alboni, à Passy; bords de la Marneà Charenton ; Montrouge, près le fort ; Gentilly ; Arcueil, chemin allant anx carrières, près de l'Aqueduc.

Commun dans tont le Midi (Alpes-Maritimes, Var, Bouches-du-Rhône, Vaucluse, Gard, Héranlt, Ariège, HauteGaronne, Lot-et-Garonne, Basses-Pyrénées), l'Helix Xalonica remonte volontiers le long des eôtes de l'Allantique (Charente, Charente-Inférieure, île de Ré, Vendée, Finislère) el même de la Nanche (Seine-Inférjeure, Pas-de-Calais); 
il s'est acclimaté à Lyon et dans I'Indre-et-Loire. Hors de France, celle espèce vit en Espagne (Servain), en Portugal (Locard) et en Autriche, Trieste (Westerlund).

\section{Helix alluvionum, Servaly.}

Ilelix alluvionum, Servain, 1880. Moll. Esp. Port., p. 102. - Locard, 1894.

Coq. terr., p. 222.

Le type de cette espèce a été découvert par le Dr Servain aux environs de Pampelune en Espagne. La coquille du square Alboni est bien lypique; de taille un pen forte, son test est assez épais, blanc-roux, crétacé, peu brillant. Diam. : 15-16 1/2 millim.; haut.: 1 1 1/2-12 1/2 millim.

Nous n'avons recueilli que trois échantillons, parfaitement adultes, de cette espèce. Ils vivaient, au milieu d'une colonie populeuse de l'Ilelix Cyzicensis, l'un sur les luzernes près le fort de Montronge, les deux autres dans le square Alboni, à Passy.

Cette Irelice espagnole, qui habite aussi le Portugal, l'Algérie (alluvions de la Seybouse, près GueIma el de l'OuedSebaou, près Dellys), la Tunisie (ruines de Carthage) et l'Autriche à Trieste, nous est conmue en France des départements suivants: Alpes-Maritimes, Var, Bouches-du-Rhône, Gard, Hérault, Pyrénées-Orientales, Lot-et-Garonne, Basses-Pyrénées, Cliarente-Infériemre, Loire-Inférieure, Vendée, Ille-et-Vilaine el Calvados. Cette espèce s'est acclimalée à Jyon depuis 187 ı.

\section{Helix Cyzicensis, Galland.}

Ilelix Cyzicensis, Galland, in Coutagne, 188 . Bassin Rhône, p. 13. - Locard, r894. Cog. terr., p. 224, fig. 295-296.

Celte espèce, si bien caraclérisée, est une des plus répandues antour de Paris; e'est aussi une de celles qui conservent le mieux son galbe et son aspect méridional. De taille nor-- 
male ou même grande, le test est jaunacé, peu brillant, orné de stries fines et serrées, et de bandes brun foncé continues ou non, subtransparentes. Diam.: 14-16 millim.; haut.: 10-12 millim. Comparée aux échantillons de Beaulien en Maine-et-Loire, où l'Helix Cyzicensis est également acclimaté, la coquille parisienne présente, sur un fond moins brillant el plus jaunâtre, des bandes beaucoup moins chaudement colorées, ce qui lient à la différence de climat des deux stations. Nous signalerons une var. minor possédantle même galbe, le même test, mais au moins un tiers plus petite. Diam.: 10-11 millim.; haut.: 8-9 millim.)

Très commun dans le square Alboni à Passy, où il vil dans tont le jardin, mais surtout smr les plantes odorantes de grande taille (Ombelliferes, Foniculum, grandes Composées, etc...) introduites dans un but ornemental, l' $H$. Cyzicensis ne se rencontre qu'exceptionnellement sur les talus des fortifications (porte de Vitry) qui ne lui offrent pas une végétation suffisante. Il faut le rechercher dans les champs, contre les talus ombreux bien exposés an soleil, etc...: Bicêtre, près le fort; Montrouge, près le fort; Choisy-le-Roi, près le passage à niveau dı P.-L.-M. ; Arcueil ; bords de la Marne à Cliarenton; Sennevières, près Nanteuil-le-IIaudouin ; Lagny; Neuilly; pare du Vésinet.

L'HI. Cyzicensis, répandu dans toute la Provence, remonte volontiers le long des côtes de l'Océan Atlantique et de la Manche. Nous le connaissons dans les département suivants: Alpes-Maritimes, Var, Bouches-du-Rhône, Gard, Pyrénées-Orientales, Lot-et-Garonne, Gironde, Charente-Inférieure, île de Ré, Vienne, Loire-Inférieure, Maine-et-Loire, Vendée, Ille-et-Vilaine, Nanche, îles Chausey, Calvados, Seine-Inférieure. Il s'est aussi acclimaté à Lyon. A l'élranger, nous le connaissons en Portugal (Locard) et à l'île Minorque(TVesterlund). 
Groupe de l'H. Mendranoi.

\section{Helix Mendranoi, Servalv.}

IIelix Mendranoi, Servain, 1880. Moll. Esp. Port., p. 105. - Locard, 1894 . Loq. terr, p, 226, fig. 297-298.

Cette Hélice espagnole, dont la présence a été constatéc en Sicile, en Algérie et en Tunisie présente, autour de Paris, un galbe bien typique, mais un test moins brillant et surtout moins épais que dans les échantillons plus méridionaux. Une bande brune, étroite, souvent obsolète, parfois réduite à des points, orne presque toujours la coquille, qui est de taille normale. Diam. : r1-13 millim.; haut. : 9-1 I millim. Une var. minor, ne dépassant pas $91 / 2$ millimètres de diamètre pour 8 millimètres de hauteur, habite les planches de clôtures de la rue Barrault, dans Paris même.

Commun, sur les talus, parmi le gazon, grimpe sur le Carduus tenuiflorus, Lin. et y reste exposé à l'ardeur du soleil : Paris, talus des fortifications, porte de Vitry, porte de Gentilly, rue Barrault; cliamps, près le fort de Bicêtre; talus à Gentilly; bords de la Marne, à Charenton; bois de Meudon; Lagny.

Nous connaissons l'Ilelix Mendranoi dans les départements des Alpes-Maritimes, du Var, des Bouches-du-Rlıône, du Gard, de la Haute-Garonne, de la Gironde, de la Cliarente-Inférienre, de la Loire-Inférienre, la Vendée, et du Calvados. Cette même forme se retrouve aussi à l'île de Ré et dans les îles de Chausey.

Helix Ganovasiana, Servals.

Ilelix Canovasiana, Servain, ı88o. Moll. Esp. Port., p. ıo6. - Locard, 1894. Coq. lerr., p. 227.

L'Helix Canovasiana est une forme hispanique qui a été

1 Sous le nom d'II. Manchanoi (per error.), Caziot, 1896 . Faune malac. Vendée, in Feuille Jeunes-natur., 3e série, XVI, p. 11, 54 et 97. 
constatée en Portugal, en Algérie, en Tunisie et en Sicile. Nos échantillons sont de galbe identique, mais de taille plus pelite; ils présentent, en outre, une coloration plus jaunacée, moins brillante, et un test notablement moins épais-crétacé. Diam. : 1 I-I 2 millim.; haut. : 83/4-9 1/4 millim.

Assez rare: Paris, rue Barrault; square Alboni, à Passy; talus à Gentilly; champs près le fort de Montrouge; bords de la Marne, à Charenton.

L'II. Canovasiana est une forme assez rare dont nous avons observé la présence dans les départements des AlpesMarilimes, du Var, des Bouches-du-Rhône, de Vaucluse, du Gard, de la Drôme, de la Haule-Garonne, de Maine-etLoire, de la Vendée. Il est acclimaté à Lyon, depuis $1871^{1}$.

\section{Helix mucinica, Bourguignat.}

Melix mucinica, Bourguignat, in Locard, 1894. Coq. terr., p. 227, fig. 299300.

Petite espèce au galbe conique un peu élevé, avec le

1 Nous signalerons encore l'espèce suivante trouvée aux environs de Paris, mais sans indication de provenance précise.

Helix Mendozæ, Servain.

Ilelix Mendozx, Servain, in Locard, 1882. Prodr., p. 145 et p. $343 .-$ Loeard, 1 S94. Coq. terr., p. 227.

Cette espèce, découverte à la Barre-de-Mont, en Vendée, par M. le Dr Servain, est assez répandue dans le département de Maine-et-Loire (Champtocé, ruines du vieux Château; Beaulieu, roehe Servière; Chalonnes, ete.). La coquille de l'Anjou est de grande taille: 9 -r 4 millim. de diamètre pour 8-12 millim. de hauteur, d'un galbe parfaitement typique; le test est assez épais, solide, très généralement unicolor, mais parfois orné de I-5 bandes brunes étroites, interrompues ou non. Une variété ninor à test orné de stries plus fortes, ne mesurant que 8-9 millim. de diamètre pour $7 \mathbf{~} / \mathbf{2}$ 8 millim. de hauteur, habite la lioehe Servière à Beaulieu.

L'H. Mendozre n'est connu que dans les régions littorales de I'A tlantique et de la Manehe, dans les départements de la Vendée, de la Loire-Inférieure, du Maine-et-Loire, du Morbihan, du Finistore et de la Manclie. Letourneux et Bourguignat l'ont également signalé aux environs de Tebourba en Tunisie. 
dernier tour subanguleux et un lest subcrétacé roux clair, parfois flammulé dans le Midi, plus pâle el monochrome dans le Nord. Le type parisien est, en général, de taille assez grêle, et d'un galbe un peu plus surbaissé que les échantillons soumis ì l'influence maritime, aussi bien dans le Midi que le long du littoral de la Manche.

Rare : Talus des fortifications, près la gare d'Orléans, à Paris; Lagny.

L'H. mucinica est une forme assez commune dans presque tout le Midi (Alpes-Maritimes, Var, Vaneluse, Gard, Aude, IIaute-Garonne): elle remonte assez haut sur les côles de l'Allantique el de la Manche, oủ nous en avons constaté la présence à Dieppe, clans le département de la Seine-Inférieure ${ }^{1}$.

\section{Helix papalis, Locand.}

Melix papalis, Locarl, 1887 . In Bull. Soc. Malacol. France, IV, p. 181. Locard, 1894. Coq. terr., p. 228, fig. 3or-3oz.

Cette petite forme, voisine de l'Ilelix Mendozx, dont elle se distingue à son galbe moins globuleux, à sa spire moins haute, à son ombilic un peu plus ouvert, etc... est rare dans la région parisienne. Nos échantillons sont parfaitement identiques, comme galbe el comme taille, à ceux du littoral océanique; lenr test assez mince, mais solide, est blanc jaunâtre, à peine brillant, orné de quatre, rarement de cinq bandes brunes bien apparentes,

liare: Sur les tiges des grandes plantes garnissant les fossés des fortifications, près du chemin de fer de Limours

1 Signalons encore pour mémoire l'Ilelix peregrina, (Locard, 1894. Coq. terr. 1\%. 229) vivant normalement dans les Alpes-Maritimes, le Var, les Bouches-du-Rhône, la IIaute-Garonne, et que l'on retrouve acelimatẻ en Vendé, à Simon-la-Yineuse (Rousseau, in coll.) 
et de l'entrée de l'Aqueduc des eaux de la Vanne, à la porte de Gentilly; planches de clôture, rue Barrault, à Paris; Neuilly.

Nous connaissons cette espèce des environs d'Avignon (Vaucluse), de Saint-Jean-du-Mont (Vendée), d'Ouessant (Finistere) et de Granville, dans la Manche.

Helix Sylvæ, Servaln.

IIelix da Sylex, Servain, in Locard, 1882. Prodr., p. ir5 et p. 342

- Sylvæ, Locard, 1894. Coq. terr., p. 228.

Cette espèce, dédiée par M. le $\mathrm{D}^{\mathrm{r}}$ Servain à M. José da Sylva e Castro, le savant naturaliste portugais, appartient encore au même groupe que l'espèce précédente. C'est une petite forme particulièrement maritime, très constante de galbe, d'allure et de coloration; nos échantillons sont parfaitement typiques. Nous ne l'avons encore rencontrée qu'à Neuilly.

Dans le Midi, nous ne connaissons cettc Hélice qu'à SaintTropez, à Marseille et à Carcassonne où elle a dû être importée; elle est répandue sur tout le littoral de l'Océan Atlantique et jusque dans la Manche: Charente, CharenteInférieure, île de Ré, Saint-Jean-du-Mont /Vendée), Nantes, Saint-Nazaire (Loire-Inférieure), Ouessant, Brest (Finistère), île de Cazambre, îles Chausey, Villers-sur-Mer, ctc...

\section{Helix pilula, Locand.}

IIelix pilula, Locard, 1894 . Coq. terr., p. 229, fig. 3o3-304.

Petite forme assez répandue, bien caraclérisée, mais de galbe plus globuleux que sur le littoral de l'Atlantique, à test blanchâtre ou jaunacé, rarement orné de plus de trois* bandes brunes, étroites, souvent avec une seule bande 
légèrement supra-carénale. Diam. : 10-12 millim.; haut. : 9-10 millim.

Rare: çà et là sur les talus; Paris, talus des fortifications, porte de Gentilly, porte de Vitry, rue Barrault; square Alboni, à Passy; champs près le fort de Montronge; Gentilly; Courberoie.

L'Helix pilula est une des formes dont le domaine géographique est le plus étendu : Alpes-Maritimes, Var, Bouches-du-Rhône, Vaucluse, Gard, IIaute-Garonne, Lotet-Garonne, Gironde, Dordogne, Charente-Inférieure, Vendée, Maine-et-Loire, Ille-et-Vilaine, Finistère, Calvados, Seine-Inférieure, Pas-de-Calais.

\section{Helix Ogiaca, Servatr.}

Helix Ogiaca, Servain, in Locard, 1882, Prodr, p. 115 et p. 343. - Locard, 889. Coq. lerr., P. 229.

Le type a été observé pour la première fois par M. le $\mathrm{D}^{r}$ Servain à l'ile d'Yeu, vis ì-ris les côtes vendéennes. Nous n'en avons récolté qu'un scul échantillon, recueilli il y a une dizaine d'années à Passy, échantillon bien conforme et conme taille ct comme galbe ì nos autres types, mais le test est un peu moins épais et les marbrures ont disparu; on ne distinguc qu'une seule bande médiane étroite et continuée au-dessus. Nous observons la même décoloration chez un échantillon provenant du Var.

Nous connaissons aujourd hui l'Helix Ogiaca : à la Seyne dans le Var, aux environs de Marseille et de Toulon, ainsi qu'aux îles Chausey, dans la Manche. Bourguignat et Letourneux l'ont signalé, en $188_{7}$, aux environs de Gabès, en Tunisie. 
Helix Scicyca, Bourgulgnat.

Helix Scicyca, Bourguignat, in Locard, 1894 . Cor. lerr., p. 230.

L'Ifclix Scicyca est une espèce essentiellement maritime, dont nous avons déjà constaté l'introduction récente à Beaulieı, daus le département de Maine-et-Loire, oì elle est bien ty pique.Le seul ćchantillon parisien que nous possédions provient du square Alboni, à Passy ; il est de petite taille, peu typique, beancoup plus globuleux que la coquille des iles Chausey et non adulte. Cette espèce ne semble pas acclimatée, car malgré nos actives recherches dans la même localité en septembre-octobre 1902 et pendant l'automne de 1903 , nous n'avons pu en retrouver un second exemplaire.

L'Helix Scicyca n'a encore été signalé que dans les départements côtiers ou soumis à l'influence maritime comme: les Bouches-du-Rhône, le Garr, le Maine-et-Loire, l'Ille-et-Vilaine, la Manche. Nous le connaissons aussi de l'île de Rè et des îles Chausey; enfin cette même espèce a èté signalée en Portugal (Locard).

Groupe de l'Il. lineala.

Helix lineata, OLIvi.

Ilelix lineata, Olivi, 1799. Zool. Adriat., p. 77. - Locard, 1894. Cor. Lerr., p. 230 , fig. 3o5-3o6.

L'Helix lineata est une forme qui, actuellement, estassez répandue autour de Paris. Elle y est généralement peu typique, le galbe s'étant assez surbaissé pour donner ì la coquille un aspect plus globuleux que réellement conique. Le test est moins épais, moins solide et moins brillant, les bandes généralement plus étroites et moins chaudement 
colorées que chez les échantillons de l'Ouest et du Midi. Diam. : $12-15$ millin,; haul. : $101 / 2121 / 2$ millin.

Cette espice vil en colonies pen populeuses, sur les tiges, le long des talus des fortifieations : boulevard Tietor; square Alboni, à Passy: cliamps et Lalus a Gentilly et près le fort de Montronge; Charenton, bords de la Marne: Semnevieres, près Nantenil-le-Haudouin.

L'Il. lineata est une forme répandue sur le littoral de la Méditerranéc el de l'Océan Mtlantique. Nons le connaissons dans les Alpes-Maritimes, à lîle Sainte-Marguerite, dans le Var, les Bonches-du Rhône, le Gard, l'Hérault, la HauleGaromne, le Gers, le Lol-et-Garonne, aux îles de Ré el d'Onessant, dans les départements de la Tendée, d'Indreel-Loire, de la Gironde, de la Charente-Inférienre, de Maine-et-Loire, du Calvados, de la Seine-Inférieure, etc. Elle habite également l'Espagne (Servain, Westerlund), le Portıgal (Locard), l'Algérie el la Sicile (Bourguignat, IVesterlund).

\section{Helix melantozona, CAricr.}

IIclix melantozona, Cafici, in Locard, is 84 . Coq. terr., p. 231, fig. 3o7-3o8.

Cette belle espèce, au galbe si caractérisé, habite le Midi et le littoral océanique de la France. Le seul échantillon que nous possédions a élé recueilli, il y a quelques années, sur les luzernes, aux environs du fort de Montrouge. Il est d'ailleurs parfaitement conforme, comme galbe et comme coloration, ì d'autres individus de l'Ouest ou du Midi de la France. Depuis lors, nous l'avons récolté assez abondamment dans les earrières d'Areucil; mais, en général, les échantillous sont de taille un peu faible, la spire tend à être moins conique, et les bandes décoratives sont moins chandement colorées. 
L'Helix melantozona est une forme peu commune, habitant le Midi de la France, et remontant le long des côtes de l'Atlantique. Nous l'avons également signalé an pied de la Roche-Servière à Beaulieu, dans le département de Maine-et-Loire.

\section{Helix Germaini, Locard.}

IIelix Germaini, Locard, 1902. Mss.

Cette espèce, qui se rapproche de l'Helix agna, Hagenmüller ${ }^{1}$, habite les talus des fortifications, près la gare d'Auteuil à Paris, et un champ près le fort de Bicêtre. Elle est très peu abondante. ${ }^{2}$ Quoiqu'elle n'ait pas été signalée

1 Hagenmüller, in Locard, 1882. Prodr., p. 116 et 344. - Loeard, 1894. Coq.terr., p. 232 .

2Helix Germaini, Locard, mss., rgo2. "Coquille d'un galbe globuleuxeonique, plus large que baut, bombé en dessous, conique légèrement tectiforme en dessus; spire à tours très faiblement convexes, à croissance un peu lente, progressive, le demier très notablement plus grand, bien arrondi, s'ovalisant à peine à l'extrémité, très faiblement déclive vers l'ou verture; suture très peu accusée; ombilic petit, profond, à peine évasé à sa naissance; ouverture oblique, assez échanerée par l'avant-dernier tour, presque circulaire ou a peine ovalaire-transverse; péristome droit, très faiblement réflèchi vers l'ombilic, aigu, avee un double bourrelet interne, passant du roux carnéolé au brun; test solide, un peu aminci, subcrètacé, d'un blane grisâtre peu brillant, muni de une à trois bande brunes étroites, infra-médianes, la bande supérieure parfois continuée en-dessus vers la suture, orné de stries fines, serrées, un peu irrégulières; sommet brunâtre. - Hauteur : 9-9 I/2 millim.; diam. : I 1-13 millim.
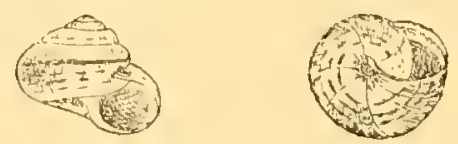

"Cetle élégante espèce doit prendre place à la suite l'II. agna, Hagenmüller, dans le groupe de l'II. lineala. Elle diffère de l'H. agna par son galbe moins conique, plus globuleux dans son ensemble, avee la spire moins baute, moins ètagée, faisant par conséquent paraitre la suture moins profonde; par son dernier tour plus haut et bien arrondi ; par son ouver- 
dans la faune méridionale, son faciès général est tel qu'il se rapproche indubitablement des formes que nous signalons et qu'il nous semble intéressant de lui faire prendre place ici, mais il nous est impossible de dire si c'est une espèce normalement méridionale acclimatée dans le bassin parisien, ou si c'est une modification profonde d'une espèce déjà existante, comme l'Helix agna par exemple. L'Helix agna n'a pas encore été signalé dans le bassin de Paris; mais nous le connaissons dans les départements du Var, des Bouches-du-lihône, de l'Hérault, du Gard, de la HauteGaronne, de la Vendée, en Algérie, en Tunisie et en Sicile.

\section{Helix fœdata, Hagensüller.}

Helix foedata, Hagenmüller, in Locard, 1882. Prodr., p. 116 et 341, - Locard, 1894 . Coq. terr., p. 232.

Nous ne possédions, jusqu'en ces derniers temps, qu'un seul échantillon de cette espèce, recueilli mort en $\mathbf{1} 898$, aux environs du fort de Montronge. Il est de belle taille (diam. : I 8 millim.; haut. : 15 millim.), bien adulte et absolument conforme à d'autres types du Midi. Nous en avons récollé tout récemment de beaux spécimens à Arcueil, près de l'Aqueduc, de très belle taille, mais avec une tendance à avoir la spire un peu moins haute, un peu moins conique que les échantillons du Midi de la France et de l'Algérie.

Nous connaissons l'Helix fodala dans les départements du Var, des Bouches-du-Rhône, du Gard, de l'Aude, de la Haute-Garonne, des Pyrénées-Orientales et, sur le littoral océanique, de la Loire-Inférieure. Celte même espèce est

ture plus circulaire et plus encochèe par l'avant-dernier tour; par son péristome ayec ses bourrelets internes multiples et distincts; par son test un peu moins épais avee des stries plus fines et plus rapprochées; etc. » 
également très répandue en Algérie (Bône, La Calle, Bougie, etc...) et en Tunisie ${ }^{1}$ (Feriana, Tebessa, etc...) [Letourneux et Bourguignat].

\section{Helix foedatina, Locakd.}

Ifelix fuedatina, Locard, 1892. Corf. terr., 1894, p. 232.

Une colonie populeuse de cette espèce habite sur les chardons, au bord de la route de Choisy-le-Roi à Créteil, tout contre le passage à niveau du P-L-M. Les individus de taille moyenne (haut.: 10 1/2-12 millim,; diam. : 12 1/2(5 milim.), sont bien typiques comme galbe; leur test blane, opaque, monochrome, subcrétacé, est beaucoup moins brillant et moins solide que chez les coquilles méridionales.

Outre la station de Choisy-le-Roi, nous signalerons eneore le Bois de Meudon.

L'Ilelix foedatina nous est connu dans les départements des Alpes-Naritimes, du Var, de Vancluse, dı Gard, de l'Aveyron, de la Gironde, de la Charentc-Inférieure, de la Loire-Inférieure, de la Manche et du Pas-de-Calais. Il vit également en Algéric el en Tunisie.

Helix Tabarkana, Letounneux et Bourguignat.

IIelix Tabarkana, Lelourneux et Bourguignat, 1887. Prodr. Malacol. Tunisie, p. 5. - Locard, 189 . Coq. terr., p. 233.

Le type à été déconvert dans l'île de 'Tabarka, en Tunisie, par M. le Conseiller Letourneux. Nos échantillous du square Alboni, à Passy, sont de petite taille, assez roisins

${ }^{1}$ Letourneux et Bourguignat ont signalé, en Tunisie, une var. major à Béjà (IIagenm.) et au Djebel Reças(Letourn.) et une var.minor à Tebourba (Letourn.) de cette espèce [Letourneux et Bourguignat, 1887 . Prodr. Malacol. Tunisie, p. 54]. 
du lype, quoique d'un galbe plus globuleux, à test mince, blanc jaunacé, peu brillant, orné de $1-4$ bandes brunes pâles et étroites.

L'Helix Tabarliana ne nous est connu que du square Alboni, où il formail, en 1898 , une colonie peu populeuse sur une touffe de Finniculum officinale, L. Depuis, les travaux entrepris pour la construction du Métropolitain ayant modifié considérablement cetle station, l'espèce a entièrement disparu.

Cette Hélice ne s'éloigne que difficilement des côtes. Nous la connaissons sur le littoral miditerranécn (Alpes-Maritimes, Var), et dans le département de la Haute-Garonne, d'où elle remonte sur les côles de l'Océan Atlantique (Vendée, Loire-Inférieure, Finistère) et même de la Manche (Ille-ct-Vilaine, Manche.)

\section{Helix Trapanica, Bertuier.}

IIelix Trapanica, Berthier, in Locard, r8y4. Cog. terr., p. 234.

Nous ne possédons qu'un seul échantillon de cette espèce, recucilli vivanl, sur la tige d'une graminée, dans un champ près du viaduc d'Arcueil. C'est une coquille de grande taille, mesurant i $7 / 2$ millim. de diamètre pour $161 / 2$ millim. de hauteur, au galbe conique-globuleux, très fortement conique en dessus et très bombé en dessous. Le test est blanc, brillant en dessus, jaunacé en dessous, finement el très irrégulièrement strié, orné de deux bandes brunes étroites, l'une supracarénale, l'autre lrès exactement carénale, toutes deux élargies au voisinage de l'ouverture.

Nous connaissons cette même forme dans les Bouches-duRhône, le Gard, l'llérault, et à l'île de Ré ${ }^{1}$.

' Une autre forme voisine, mais de taille plus petite, l'I. didymopsis, P. Fagot (in Locard, 1882 . Prodr.. P. 116 et 345. - Locard, 1894 . Coq. terr., 


\section{Genre Cochlicella, Risso.}

\section{Cochlicella barbara, Lixí.}

Helix barhara, Linné, i 758 . Syst. Nat., éd. X, p. $77^{3}$.

Cochlicella acuta, Locard, 1894 . Corf. terr., p. 23s, fig. 32 I.

Le Cochlicella barbara a été plusieurs fois recueilli aux environs de Paris. Bourguignat l'a autrefois récolté dans Paris même, au quai de Javel ${ }^{1}$, et M. Mabille en a trouvé de très beaux échantillons dans les îles de la Seinc, un peu au-dessous du viaduc d'Auteuil. Ce sont là des faits exceptionnels, et cette espèce, sans doute apportée avec les bateaux marchands faisant le service du Havre, n’a pu se maintenir. Elle a également disparu des environs de Lyon où l'un de nous l'avait récoltée en ${ }_{1} \$_{7} 6^{2}$.

Le Cochlicella barbara est une coquille essentiellement maritime et subcosmopolite que l'on rencontre aujourd'hui sur tout le littoral maritime français.

p. 234, fig. 309,310$)$ a élé récemment observée par M. Rousseau à SimonJa-Vineuse en Vendée; on ne la connaissait que dans les Alpes-Maritines, le Var, l'Aude, l'Hẻrault et la Dordogne.

1 Locard (A.), i 882 , Contrib., IV, p. I4.

${ }^{2}$ Locard (A.), 1882. Loc. cit., p. 14. 


\section{CONCLUSIONS}

Voici donc toute une faunule, comprenant une cinquantaine d'espèces, appartenant normalement à la faune méridionale et qui, cependant, ont toutes été recueillies aux environs de Paris. Quelques unes ont été récoltées mortes et en petit nombre, mais la grande majorité vivent en colonies populeuses, dans les localités que nous avons signalées dans la première partie de ce travail. Ces introductions sont certainement de date récente, aucun des anciens auteurs, Geoffroy ${ }^{1}$, Poiret ${ }^{2}$, Brard ${ }^{3}$, Pascal ${ }^{4}$, ayant traité de la Malacologie parisienne, u'en ayant fait mention. En i 87o, M. Mabille ${ }^{5}$ ne pouvait encore recueillir que quelques rares Helix ademata dans la Plaine Saint-Denis et, en ${ }_{1} 878$, II. le $\mathrm{D}^{\mathrm{r}}$ Jousseaume ${ }^{6}$ ne signale que les $H$. variabilis et II. Pisana.

1 Geoflroy, Traité sommaire des coquilles tant fuviatiles que terrestres, qui se trouvent aux environs de Paris, Paris, $176_{7}$, in-12, 144 p. Ouvrage parfois accomparné de 3 pl. gravées par Duchesne. - Traduction allemande par Martini. Nüremberg, 1767 , in-8.

2 Poiret (J.-L.-M.), Coquilles fluviatiles et terrestres observées dans le département de l'Aisne, et aux entirons de Paris, Prodrome, Paris, avril 1809 , in- $12,119 \mathrm{P}$.

3 Brard (C. P.), llistoire des coquilles terrestres et fuviatiles qui vivent all $x$ environs de Paris, Paris et Genève, 1815 , in-12; 10 pl. color.

4 Pascal, Catalogue des. Mollusques terrestres el des eaux douces du département de la IIaule-Loire et des environs de Paris, Paris, 1873 , gr. in-8, 8 r p.

5 Mabille (J ), I $8 ; 0$. Ilisloire malacologique du bassin parisien ou histoire naturelle des animaux Mollusques terrestres el fuviatiles qui vivent aux

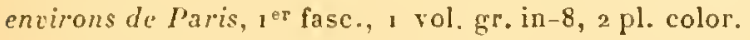

6 Jousseaume (Dr), Faune malacologique des environs Paris, In Bull. Soc. Zool. France, 1876 à 1881 , in- 8 , a vec $\mathrm{pl}$. 
C'est, comme on le voit, une modification profonde et récente dans l'allure normale, autochtone de la faune du bassin parisien, et dont il y aura désormais lieu de tenir compte lorsqu'un nouveau catalogue en sera dressé. Toutes ces espèces sont-elles définitivement acclimatées; cela nous parait très probable, au moins pour la plupart d'entre elles; en elfet, il en est quelques-unes, sans doute parmi les plus anciennement introduites, qui ont pu donner naissance à des colonies très populeuses, montrant bien qu'elles ont eu ì subir les rigueurs de plusieurs hivers consécutifs. Ajoulons que bien certainement cette liste est loin d'être close, et qu'avec le temps de nouvelles observations permettront de l'accroître encore ${ }^{1}$.

Examinons maintenant dans quelles conditions cet élément nouveau de la faune parisienne se présente, et quelles conclusions on est en droit d'en tirer.

Nons observerons que notre faunule introduite pent être groupcé en plusieurs sections dont chacune correspond à une station constituant un milieu gréographique différent. Nous étudicrons d'abord, avec quelques détails, chacune des stations principales.

$1^{\circ}$ Bonds de la Marine (rive droite), extre le confluent ET Jonville-1.E-Pont. - Celle station offre des particularités topographiques très spéciales. Le cours de la Marne, encom-

1 C'est ainsi qu'il faut probablement ajouter à cette liste le Zua Locardi, Pollonera (Pollon., 1885. Moll. Piem., p. 21) que nous ne connaissons que dans le Sud-Est de la France et en Italie, et que M. le marquis de Monterosato nous a adressé toul récemmenl, comme ayant été trouvé dans le bois de Boulogne. 
bré de nombreuses îles boisées offre, sur la rive droite, des berges garnies d'une végétation luxuriante. A une vingtaine de mètres de la rivière court un talus élevé, séparant la Marne du canal latéral, et contre lequel pousse, sur le rebord sud, une riche végétation composée de nombreuses graminées:(Poa, Festuca, clc...) entremêlées de touffes de Rubus, Potentilla, Galium, Rumex, Urtica, etc., etc.. C'est au milieu de ces plantes que vivent nos Mollusques introduits, d'ailleurs fort nombreux en espèces: Ilelix Pisana, II. Pisanella, H. Cuttati, II. Mendranoi, II. Avenionensis, H. nemausensis, H. Talonica, II. Canovasiana, II. lineata, II. Mendranopsis, II. limbifera, H. acomptiella, H. leviculini, etc...

Les Variabiliana restent assez nettement cantonnés contre la levée faisant face à la Marne, mais les espèces du groupe Jisana ont rayonné et envahi les berges de la rivière où elles vivent au milieu des taillis les plus épais, en compagnie des $H$. nemoralis, $I$. arbustorum et de nombreux Succinea. Toutes ces espèces conservent un galbe très constant et voisin de celui qu'elles affectent dans le Midi. Ce fait tient, sans aucun doute, anx conditions exceptionnelles de la station : le talus, bien exposé au midi, couvert d'une riche végétation et entretenu frais par le voisinage immédiat de la Marne est un milieu évidemment favorable au développement de nos Mollusques qui trouvent là un nıodus vivendi beaucoup plus avantageux pour eux que celui des talus arides, brûlés par le chaud soleil de Provence. Cette station nous rappelle de tous points celle déjà signalée par l'un de nous aux environs de Lyon ', sur les bords du Rhône, entre le Pare de la 'Tète-d'Or et le fort de la Vitriolerie, station où abondent les Variabiliana qui, déjà introduits en

1 Locard (A.), 1882. Contrib., IV, P. 11. 
ı840, ont pris tout à coup, entre 1880 et 1882 , un remarquable développement.

Le mode d'introduction des Hélix de Charenton nous est parfaitement connu, au moins pour quelques-unes des espèces que l'on y rencontre; nous en avons fail l'histoire en traitant, dans la première partie de ce mémoire, l'H. Pisana; remarquons simplement que, très probablement, avec les $I I$. Pisana rapportés du Midi et jetés pêlemêle contre le talus de la Marne, se tronvaient quelques échantillons de formes affines qui ont fail souche et essaimé ensuite sur les talus, parallèlement aux Pisana.

$2^{\circ}$ Cholsi-le-Rol, route de Crétell, prés le passage a niveau du Chemix de far P.-L.-M. - La route qui, partant du pont de Choisy-le-Rioi, conduit au chemin de fer du P.-L.-M., est bordée de talus assez élevés, dont l'un, exactement exposé an midi, sert d'asile à de très populeuses colonies de Trariabiliana. Leur distribution est des plus intéressantes. Extrêmement abondants sur le talus sud, avant, mais surtout après le passage à niveau, ils deviennent de plus en plus rares si l'on s'éloigne de ce point précis : à quelques centaines de mètres, ils ont entièrement disparu ${ }^{1}$. La végétation de cette localité, assez luxuriante, offre surLoul, outre de nombreuses graminées, des Artemisia et des Carduus, plantes sur lesquelles nos Helix se cantonnent de préférence. Nous avons pu voir un espace de plusieurs mètres carrés où poussaient exclusivement des chardons el littéralement envahis par les Variabiliana, à tel point que le sol disparaissait sous une couche blanche forméc par les coquil-

1 En réalité, on trouve de loin en loin, en revenant vers Choisy-le-Roi. quelques rares Melix, mais il est impossible d'en recueillir un seul individu sur la route de Maisons-Alfort, située à moins de roo mètres du passage à niveau, mais exposée au Nord. 
les. A mesure que la saison s'avance, les Helix qui, l'été, habitent les flancs du talus et le rebord sud de la route, descendent et se cantonnent an pied du talus, mieux abrité des vents.

Les espèces sont assez nombreuses; parmi les plus répandues, nous citerons : Helix ademata, H. Cyzicensis. I1. Mendranopsis, H. foedatina, H. fera, H. Guideloni, II. Avenionensis, H. Nemausensis, H. limarella, II. acomptiella, II. ericelorum, etc.

Une particularité très remarquable chez des espèces introduites, et qui tient à la situation très favorable de la station, est la présence de var. major chez certaines formes comme II. Cyzicensis, H. Mendranopsis, H. limarella. Cette dernière espèce surtout, nous a fourni une forme major plus grande que toutes celles que nous connaissons du Midi.

Le mode d'introduction de cette colonie ne saurait être, pour nous, douteux; son area l'indique suffisamment; il faut l'attribuer au chemin de fer : les nombreuses marchandises qui arrivent du Midi par le P.-L.-M, expliquent suffisamment leur présence et le voisinage de l'important dépôt de Villeneuve-Triage ne fait que fortifier notre opinion.

30 Squalie Alboni a Pamis (Passy). - Ce square, de plantation récente, est établi sur un terrain fortement en pente dominant la rive droite de la Seine. Relativement accidenté, bordé de hautes constructions, élevées au moment de l'Exposition de r 900 , qui le mettent à l'abri des vents, ce square sert d'asile à des colonies très populeuses de Variabiliana, qui, parfois, ont completement envahi les arbustes et les hautes Composées. On pent y observer la grande préférence de ces Mollusques pour ces hantes Composées et surtout pour les Foniculum, préférence que l'un de nous a déjà signalée à propos des espèces de l'Anjou ${ }^{1}$.

1 Germain (L.), 1903, Et. Moll. Maine-el-Loire, p. 19 et p. 125. 
Les Variahiliana du square Alboni, en général de grande taille et bien typiques, sont nombreux en espèces et vivent en compagnie de riches colonies de grandes striées. Nous citerons notamment :

Helix Cyzicensis, H. fera, II. Nemausensis, H. Avenionensis, H. Mendranopsis, H. Guideloni, H. Talonica, II. Canovasiana, H. acomptiella, H. lineata, H. Grannonensis, II. pilula, H. papalis, H. scicyca, H. alluvionum, elc.....

Le mode d'introduction de ces espèces nous est également counu : nous verrons plus loin que les talus des fortifications nourrissent de nombreux Variabiliana, et nous dirons comment, selon nous, ils ont dî être introduits. Or, de l'autre côté de la Seine, en face le square Alboni, la gare de marchandises de Grenelle-Ceinture a amené de nombreuses Xérophiliennes qui ont rayonné jusque sur les quais herbeux de Javel. La construction du chemin de fer de l'Ouest (gare Saint-Lazare aux Invalides) a établi la jonction entre les deux rives du fleuve et nos espèces, suivant le chemin de fer, se sont peu ì peu répandues, d'abord dans l'ile des Cygnes, ensuite dans le square Alboni. Ajoutons que les travaux, entrepris pour la ligne métropolitaine $n^{\circ} 3$, ont déjà notablement modifié l'aspect de cette station : la partie centrale du square, qui servait d'asile à de nombreuses colonies a été éventrée, el nos coquilles ont dî se réfugier vers le mur d'enceinte de la rue des Eaux.

4. Sennevières, près Navteull-te-Hatinouin (Oise). Nous ne reviendrons pas sur celle station que l'un de nous a étudiée avec tous les détails que comporte un tel sujet'. Bornons-nous ì rappeler ici que, dans cette localité oì la

${ }^{1}$ Locard (A.), 1805 . Nolices conchyl, XXXY, Nouv. stat, esp. méridion., elc. in L'Echange, XI, p. 121-122. 
faune malacologique est si pauvre, une riche colonie de Variabiliana s'était cantonnée exclusivement sur le talus sud d'un fossé bordant à droite la route conduisant de Sennevières à Chevreville. Les espèces, assez peu nombreuses mais bien typiques, sont : Helix variabilis (A. R.), $H$. Cyzicensis (C.), II. lineata (C.), H. Mendranopsis (C. C.) et $I I$. acomptiella (A. C.) qui vivaient en compagnie d'une colonie médiocrement populeuse d'H. ericetorum de petite taille. Quant au mode d'introduction de es espèces, il est des plus curicux : il faut l'attribuer à des nomades qui, ayant stationné avec leur roulotte dans cet endroit désert et éloigné de tout centre de population, ont laissé là quelques jeunes Mollusques qui ont fait souche et semblent s'être acclimalés définitivement dans le pays.

5. Carriéres d'Arcueil. - Si quittant, à Arcueil, l'avenue de la Liberté, on se dirige vers les carrières, on pénètre bientôt dans un chemin bordé de talus qui, d'abord élevés et escarpés, ne tardent pas à s'abaisser presque au niveau de la route. C'est contre ces talus, et surtout contre l'un d'eux parfaitement exposé au Midi, que vivent, au milieu d'une belle végétation composé de Graminés, de Chardons, de Centaurées, de Rubus, etc., de populeuses colonies de Variabiliana qui se sont aussi un peu dispersées sur la lisière des champs qui dominent le chemin. Les espèces y atteignent une taille voisine de celle qu'elles ont normalement dans le Midi, leur galbe ne subil que des modifications légères et leur test, un peu brillant, est assez richement décoré. Nous y avons récolté quelques espèces fort intéressantes, comme par exemple : l'H. Trapanica, forme rare du littoral médilerranéen; l'H. melantozona, gardant son galbe élevé, etc. On recueille encore, en plus ou moins grande abondance: Helix leviculina, H. Nemau- 
sensis, H. Avenionensis, H. Grannonensis, H. fera, H. terraria, H. limbifera, II. subtassyana, H. mendranopsis, H. Cyzicensis, H. Kalonica, II. alluvionum, H. Salentina, 11. acompticlla, II. lineata, HI. fodata, etc.

Cette eolonie est en pleine prospérité et son introduetion ne doit pas remonter à plus de huit ou dix ans, au dire des agriculteurs des environs que nous arons pu interroger. Sans aueun doute, ces Variahiliana ont été apportés avee les détritus des Halles employés pour fumer les cultures. Ils se sont alor's dirigés vers les talus de la route, beaueoup mieux abrités que les ehamps environnants. Certaines années, d'après les cultivateurs, les coquilles sont beaucoup plus abondantes et atteignent la taille du pouce. Nous avons pu d'ailleurs observer que les formes élevées ou de grande taille (Helix fodata, 11 . melantozona, $H$. Salentina, $H$. acomptiella, etc.) se localisent sur les talus de la partie la plus eneaissée de la route; un peu plus loin, les ehamps étant presque au niveau du ehemin, on ne rencontre plus guère que les petites espèces si répandues dans tonte la banlieue parisienne (Hclix fera, II. Grannonensis, II. Nemausensis, etc.).

6. Talus des fortifications de Paris lit réglons avoisiNantes. - Tout autour de Paris on pent observer, en plus oll moins grande abondance, suivant les loealités étudiées, de riehes colonies de Variabiliana vivant sur les talus herbeux des fortifieations. En comparant les écliantillons recueillis avee eeux des localités que nous venons d'étudier, on observe en général d'assez grandes différenees : les coquilles des fortifieations étant plus petites, moins chandement eolorées, ce qui tient évidemment à l'aridité plus grande de la station. Iei, sauf de rares exceptions, pas de taillis ni d'arbustes, mais un simple gazon, exposé en 
plein soleil, parsemé de rares Chardons et de nombreux pieds de Centaurea calcitrapa, L., que nos Helix envahissent avec avidité. Les espèces sont fort nombreuses, et si quelques-unes se localisent volontiers (comme par exemple l'H. Kalona près de la gare d'Orléans), la plupart sont partout très répandues. Nous citerons notamment:

IIelix ambielina, H. mucinica, H. limarella, H. suberis, H. Mendranoi, H. Cyzicensis, H. fera, H. Guideloni, H. Avenionensis, II. Nemausensis, II. Malonica, II. acompliella, II. Canovasiana, II. Jusiana, H. Mendranopsis, II. Grannonensis, H. pilula, H. papalis, H. lineata, II. limbifera, II. Germaini, etc., etc.

En dehors des fortifications, la grande majorité de ces espèces se retrouvent dans toutes les communes de la banlieue parisienne : elles abondent à Ivry, Gentilly, Bicètre, Arcueil, Montrouge, Malakoff où elles ont rayonné jusqu'au delà du plateau de Châtillon ${ }^{1}$ !., à Issy-les-Moulineaux, Boulogne, etc. Elles sont moins répandues dans la banlieue nord et du côté de la plaine Saint-Denis, mais on les retrouve aboudantes à Nogent, au Perreux, à Joinville, à Charenton, et mème à Vincennes où elles sont beaucoup plus rares et n'existent pas dans le bois.

Dans toutes ces localités, les Variabiliana recherchent les talus abrilés et exposés au Midi; sur telle route oì ils abondent sur le talus sud, il sera impossible d'cu trouver un seul écliantillon sur le talus nord. Ce fait est des plus caractéristiques, et nous avons eu maintes fois l'occasion de le constater. Mais en outre, il arrive que, presque toujours, ces colonies nouvelles, même lorsquelles devienuent populeuses, sont très localisées, soit parce que leurs éléments constitutifs n'ont pas encore eu

1 Le plateau de Châtillon a surtout donné asile à l'H1. fera. 
le temps de se disperser, soit parce qu'elles éprouvent quelques difficultés à retrouver plus loin un milieu aussi propice à leur développement.

Les causes très multiples qui président à de telles migrations peuvent se grouper sous deux chefs principaux : causes naturelles, causes aecidentelles.

Les Mollusques se déplacent parfoís lentement, suivant de préférence les lignes isothermes, et gagnant de proche en proche, se fixent plus ou moins loin de leur liabitat normal, ainsi que nous aurons occasion de le voir plus loin. Si de telles migrations peuvent expliquer la présence d'espèces méridionales sur certains points, on ne saurait s'en contenter pour la banlieue parisienne. C'est encore à ces mêmes causes que l'on peut attribuer l'extension lente et progressive d'un grand nombre de formes malacologiques normalement méridionales et qui pourtant, sous l'influence bienfaisante du gulf-stream, ont pu remonter tout le littoral océanique et même les bords de la Nanche, jusque dans le Pas-de-Calais, franchissant des cours d'eaux comme la Gironde, la Loire, la Seine. Il est du reste ì remarquer que plus on s'éloigne du centre d'origine, plus le nombre des espèces ainsi dispersées devient restreint, tout en donnant lieu parfois à des colonies très populeuses, lorsque la nature des milieux vient à favoriser les conditions de développement de ces espèces.

Les causes accidentelles sont fort nombreuses : en dehors des déplacements volontaires on tentatives d'acclimatation faites par les naturalistes eux-mêmes, et dont il nous serait 
facile de citer de nombreux exemples ${ }^{1}$, il faul surtoul tenir comple des apporls faits, soil avec les fourrages, soit avec les légumes importés en si grande quantité du Midi depuis quelques années, soil avec lout autre véhicule.

C'est ainsi que l'II. Pisana, qui a si bien prospéré à Charenton, doil son acclimatement, ici involontaire il est vrai, a la main de l'homme. Mais la grande, l'immense majorité des Variabiliana introduits aux environs de Paris, l'ont élé avec les marchandises transportées par ehemin de fer. Depuis quelques années surtout, les légumes du Midi de la France, de l'Algérie, de l'Espague sont expédiés aux halles de la capjale en quantités considérables : ces légumes, les feuilles de salade notamment, donnent facilement asile à de nombreuses coquilles jeunes, pent-être même à des oeufs qui, rejetés avec les déhris maculés el invendables, ont rapidement prospéré el donné naissance i de nombreuses colonies ${ }^{2}$. li importe d'ailleurs de remarquer que ces introductions ne viement pas uniquement du Midi, mais encore arec les nombrenx légumes exportés de l'Onest de la France, de la Normandie, de la Bretagne on de l'Anjou. Les coquilles ainsi transportées, ayant déjà subi un premier acclimate-

1 Nous rapporterons ici un curieux fait d'acclimatation de Hollusques du fait volontaire de la main de l'homme. En r 883 , II. Roy, cultivaleur au Moulin-à-Vent près Lyon, trés épris de toutes choses touchant à l'histoire naturelle, avait làché dans son jardin un petit lot d'llelic Lucorum (Linné, ${ }_{7} 758$. Syst. nat., éd. X, p. 773 ); cette espece vit comme on le sait, dans l'ltalie septentrionale et centrale, dans le Piémont, la lombardie, la Toscane el jusquen Vénétie, jouant dans ees pays le rúle de notre $I$. pomalia. Malgré la différence de lalitude, es $I$. Lucorum se sont développés en telle abondanee, quà diverses reprises $\mathbf{~ l l}$. Roy a dü leur faire la chasse. Malgré les rigueurs de plusieurs hivers survenus depuis vingt ans, la colonie continue à être des plus prospéres. Lne telle observation peut aroir son importance au point de vue de l'élevage et de la domestication des eseargots.

${ }^{2}$ C'est ainsi qu'ont été introduits les $I I$. Pisana des environs de Lyon. Locard (1.), 1881. Tarial, Malacol. Bassin Rhönc, t, 1I, p, 130.

A. L. 
ment sur les côles, sont plus robustes, supportent beancoup mienx leur nouvel habital et restent toujours plus typiques.

De l'enquête à laquelle nous nous sommes livrés, aux Halles mêmes de Paris, il résulte que les détrilıs de tontes sortes qui encombrent les chanssées après la vente en gros du matin, dite du carreau, sont transporlés d'abord sur les quais de la Seine, à Javel. Ce premier service a surlout lieu le malin vers 9 heures; un denxième service, vers 4 heures après-midi, n'apporte guère que les résidus des marchés de quartiers.

Aussitôl, ees détritus (feuilles maculées de choux, de salade, légnmes el fruits invendables ou avariés, pailles et enveloppes de loutes sortes ayant servi à l'emballage des légumes exportés, etc.), sont chargés sur les wagons de la Compagnie de l'Onest el d'abord garés à Crenelle. De là ils sont dirigés dans toutes les localités de la banliene parisienne où ils sont rendus comme engrais anx cultivaleurs et maraîcher's. Dès lors, les jeunes Mollusques qui étaient restés an milieu de ces débris reprennent après avoir, ainsi que nous le verrons plus loin, subi un temps d'arrêl, le cours interrompu de leur développement normal, surtont s'ils ont élé déversés dans un milieu propice; les uns se développeront sur place, d'autres iront chereher un habilat sufísamment abrité ct bien à leur convenance; qu'il vienne ensuite une série d'hivers progressivement rigoureux el l'acelimatement sera défmilif. D'ailleurs l'afflux continu de légumes dont les débris sont invariablement expédiés en des points fort éloignés de la banlieue parisienne el répandus sur les sols les plus diver's favorise singulièrement l'introduction des Variabiliana el étend constamment leur aire de dispersion, la disparition d'une colonie élant rapidement compensée par l'apparition sur un aulde point, d'une nouvelle colonie plus prospère. Enfin, les chances de propaga- 
tion sont encore accrues par ce fail que les légumes du Midi entrent surtout à Paris pendant la belle saison.

Les données précédentes expliquent également la distribution lopographique si curieuse des Tariabiliana au lour de Paris. On les observe en effet, abondamment tout le long de voies ferrées (et, en particulier, dans l'aris même, sur les talus des fortifications oì évidemment nos Helix ont suivi le chemin de Ceinture), d'où ils ont ensuite essaimé dans les terrains voisins, à mesure que l'on ulilisail les engrais apportés par le chemin de fer.

Mais si tel a été, dans l'immense majorité des cas, le mécanisme de l'introduction, de l'acclimatement et du rayonnement des espèces méridionales dans la région parisienne, il n'en est pas moins vai, qu'exceptionnellement, cet acclimatement el ce rayomement ont pu avoir d'autres causes. Parfois nos Mollusques déjà acclimatés se sont déplacés lentement d'eux-mêmes'; d'autres fois les oiscaux ont trausporté avec eux, pour la construction de leur nid ou pour lout autre motif, des brindilles où adhéraient de jeunes coquilles : celles-ci ont formé de nouvelles colonies qui n’ont pas tardé à prospérer. Dès 186 io, Lea avail observé un fait analogue : cet auteur vil se développer, dans une citerne, des Physa gyrina Say, dont les ceufs avaient été apportés par des oiscaux ${ }^{2}$.

1 Nous citerons le bel exemple suivant de déplacement lent que nous avons puobserver dans nos environs. Les bords de la Marne, entre la station des bateaux à vapeur du pont de Charenton ct les moulins d'Alfort is 5 ou 600 mètres plus bas, sont tellement fréquentés le dimanehe par les promeneurs, que l'berlue est entièrement foulée, les talus dénudés, ete. La colonie de Variabiliana s'est lentement déplacéc $\mathrm{ct}$, aujourd'hui, clle prospère à I kilomètre plus bas, au milieu d’une végétation beaucoup plus luxuriante et dans une localité bien moins fréquentée. Ici, une influence que l'on pourrait appeler dynamique a obligé la colonic à un déplacement lent, mais continu.

${ }^{2}$ Lea, Proceed. of the Acad. of Sc. of Philadelphia, mai 1860 ; lir. à prat, Pliladelphie, I862, P. 11. 
Dans une vaste excavalion ereusée aux environs de Lyon et remplie d'eau de pluie ou d'infiltration, on a vu se développer de nombreuses coquilles de Physa acula et de Limnea limosa provenant sans nul donte d'oufs ou de jeunes transportés des mares voisines par les oiseaux ${ }^{1}$. Le plus bel exemple de transport de ce genre que nous connaissions est celui du Limnca raphidia, espèce de Dalmalie retrouvée daus le lae de Silan, près de Nantua. Quelque oiscau migraleur a sans doute un jour apporté avec lui, fixé ì ses palles, un paquel d'oufs qui, déposés dans les eaux du lae, out prospéré el donné naissance à une petite colonie de celte Limnée étrangère ${ }^{2}$.

Les fourrages souvent transportés par les soins de l'administration militaire ne sont pas toujours étrangers à de telles introductions. A Lyon, loute une petite faunule méridionale a élé introduite en $1870-1871$, avec des fourrages destinés aux grandes caserues de la Part-Dieu ${ }^{3}$. Aux environs d'Ardenay dans la Sarthe ${ }^{4}$ el de Champlocé dans le Maineet-Loire", le Cochlicella barbara a été, selon tonte vraisemblance, apporté avec les fourrages militaires et, s'il ne s'est pas colonisé dans la première localité, il s'est parfaitement

1 Locard (A.), 188ı. Variat. Malacol. Bassin Thône, t. 11, p. I 40.

Les Oiseaux apportent, souvenl de fort loin, des eoquilles absolument étrangères au pays. Tel est le cas de ce Triphoris perversa recucilli à Allevard, clons l'Iscere, par M. H'alsan. [Locard (1.), 1881. Loc. cit., 1. 11, p. 138 ].

${ }^{2}$ Locard (A.), 1892 . Infl. milieux var. Malacol., p. 10 s.

${ }^{3}$ Locard (A.), 1882. Contrib., IV. p. 21.

Hême dans les fourrages comprimés, on trouve parfois des coquilles

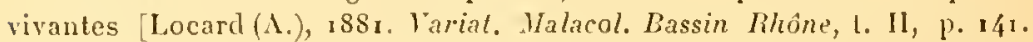
Nous arons recueilli à $\Lambda$ gers, chez un marchand de produits d'Espagne et d'Algérie, de mannifiques échantillons d'II. arenarum, Brg. et d'Il. lineala, OI. parfaitement vivants.

4 Morin (P.), 1883. Note rév. Noll. Sarthe, in Bull.Soc. Agr. Sc. Arts Sarthe, XXIX, p. \{02. - Morin (P.), 1891. Essai faun. malacol. Sarthe, p. 36.

5 Germain (L.), igo3. Etude Moll. Maine-el-Loire, p. 129. 
acclimaté dans la seconde ${ }^{1}$. Un autre exemple curienx nous est fourni par l'llelix Numidica, espèce africaine qui, introduite par des navires chargés de céréales, a d'abord été constatée an Châleau-d'If el à Montrerlon près de Marseille? De là celte coquille, grâce au climat favorable, n’a pas tardé à se propager el aujourd'hui, sans être commune, on peul la recueillir assez abondamment autour de Marseille, d'Aixen-Provence, elc... C'est encore à une intervention loute fortuite probablement d'un navire chargé de marchandises on de poissons, que l'on doit l'introduction, aux environs de Quimper, de l'Ilelix Quimperiana, forme maritimopyrénéenne que l'on ne connaîl dans aucune station intermédiaire.

D'autre part, l'extension considérable prise dans les grandes villes par les gares de marchandises constitue un excellent facteur de propagation. A Paris, anx gares d'Orléans, de Lyon, du Montparnasse, les wagons chargés de denrées provenant du Midi ou de l'Ouest séjonrnent tout contre les talus des fortifieations. Evidemment, comme l'un de nous l'a observé à Lyon, dans des conditions identiques ${ }^{3}$, les Mollusques jeunes ou leurs cufs fixés sur des détritus de toutes sortes, apportés par la main de l'homme, roulés par les vents, véhiculés par les oiseaux ou les pieds des animaux, arrivent facilement aux talus voisins el s'y propagent plus ou moins rapidement.

Mais de tels faits n'ont élé, antour de Paris du moins, que l'exception el c'est à l'utilisation comme engrais des détritus el légnmes avariés des Halles, expédiés en tous les points de la banlieue parisienne que nous devons l'existence d'une

- Signalé dẻs 18 r.3 par Millẹt, sur les ruines du vieux château de Champ-

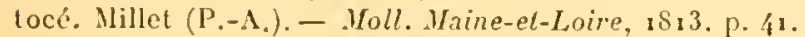

2 Bourguignat (J.-R.), s86o. Malacol. Chntean d'If, 1'. 17.

3 Locard, I882, Contrib., IV, p. 21 . 
telle faunule. Ici encore, l'homme s'est ehargé, involontairement il est vrai, d'acclimater en moins de trente années près de cinquante espèces de Mollusques absolument étrangers à la faune du pays.

Malgréle nombre de formes que nous avons eu à signaler, les espèces introduites autour de Paris constituent un groupe bien délimité. Nous n'avons en effet, rencontré ancune coquille appartenant aux groupes des Helix trepidula, Panescorsi, cespitum, etc. Cependant sur le lilloral de l'Allantique, l'Helix spherita, Hartmann ${ }^{1}$. du groupe de l'II. Panescorsi, Ber. ${ }^{2}$, remonte jusqu'à Locmariaker dans le Morljihan ${ }^{3}$ el l'II. cespilum rencontré it Lyon ${ }^{4}$ se retrouve sur les côtes océaniques jusque dans le Morbihan ${ }^{5}$, sous une forme un peu différente, il est vrai, constituant 1'II. Armoricana, Bourguignat ${ }^{6}$. Ce même II. cespitum est., ainsi que l'a dit Gassies ${ }^{7}$, fort bien acclimaté dans lout le Sud-Onest et se retronve un peu partout sur le littoral entre la Gironde el la Loire.

L'acclimatement des Mollusques n'a pas lieu partout avec

1 llartmann, 1840. Erd. und Sucssew. Gaster., I, p. 147, pl. 46, fig. 4-6.

? Berenguier, is83. Malacol. Var. Ad., p. 4.

${ }^{3}$ Bourguignat (J.-R.), 1860. Malacol. terr. el fluv. Bretagne, p. 58.

1 Locard (A.), 1882. Contrib., IV, p. 9 .

L'II. cespitum récollé à la Mouche, à Lyon, en octobre $188_{2}$, mesurait 24 millim. de diam. et 15 millim. de lıuteur, e'est-d-dire qu'il atteignait la taille ordinaile des individus du Var et des Alpes-Maritimes.

5 Bourguignat (J.-1i.), 1860 . Malacol. terr el fluv. Bretagne, 1. 57.

- Locard (A.), 1882. Prodr. Malacol. franc. p. 100 et p. 327.

- Gassies, 1880 . Des eauses de disp. cert. esp. Moll. terr. dans la Garonne et de l'acclimat. de cert. autres, in Bull. Soc. Borda, ze trim. (tir. a part, p, 7 . 
la même facilité : il dépend généralement d'un ensemble de circonstances assez diffieiles ì préciser et tient souvent aussi à la nature même de l'espèce introduite. Quelques coquilles se naturalisent facilement : tel est le eas du Sténogyre [Stenogyra (Opeas) Goodalli, Miller] de la Guadeloupe qui, introduit d'abord en Angleterre aux environs de Bristol, s'est propagé aux environs de Manchester et de Londres, notamment dans le square de Kiensington-Palace, sur les plantations de pins'. Telles sont encore les Stcnogyra (Opeas) octonoides, Adams, originaire des Antilles el de la Guyane; enfin Stcnogyra (Spiraxis) venusta, Morelet, espèce découverte en 1860 par Eudel, à Saint-Pierre de la Réunion introduits arec les plantations dans les serres du Musemm de Paris et qui s'y sont parfaitement développés, comme l'a montré M. Daulzenberg².

D'autres espèces disparaissent au contraire rapidement dès que l'on tente de les acclimater. Si les Chartreux des environs de Metz ont pu, dans le seul but de les utiliser pour l'alimentation, introduire el en quelque sorte culliver l'II. aspersa qu'ils importaient du Dauphiné ${ }^{3}$, si Gassies ${ }^{4}$ a réussi à acclimater, au moius partiellement, des Leucochroa candidissima rapportés d'Oran et de Boghar (Algérie), dans la propriété de Bel-Air près d'Ageñ ${ }^{5}$ M. Mabille a vu ce même Lcucochroa disparaître entièrement des environs de

\footnotetext{
1 Gray, in Turton, Manual, $2^{*}$ éd., 1840. - Bourguignat (J.-R), I877. Deux noureaux genres algériens, ctc., p. 68, n 26 .

2 Daulzenberg (P.), I896. Recherches Zool. sur serres Museum Paris, $\checkmark$, Mollusques, in Feuille J. Natural., rer avril, no 3o6, p. 114.

${ }_{3}$ Puton, 8547 . Moll. Vosges, p. y (note en bas de la page).

4 Gassies, I8So. Lac, cil., in Soc. Borda, à part, p. 8.

${ }^{5}$ Les Leucochroa ainsi introduits sont de taille médioere el "éprouvent, assez généralement, une tendanee au scalarisme, a la disjonclion des sutures, el il n'est pas rare de reneontrer des individus dont les tours sont presque lous élevés en rampe d'esealier. "(Gassies, loc. cil., p. 8.) Nous avons observé le même phénomène dans deux belles colonies de Leuco-
} 
Jaulgonne (Aisne) oì ce savant avait tenté son introduction'. Les nombreuses coquilles de Rumina decollata que l'on avait, à deux reprises différentes, répandues dans un jardin des environs de Lyou ne se sont pas maintenues; au bout de deux ans il n'en restail plus trace?

Nous pourrions multiplier ces exemples; citons seulement en terminant ce fait des plus curieux : un grand nombre d'espèces européennes ont été introduites aux Etats-Unis de l'Amćrique du Nord el font maintenant partie de la fame du pays, notamment Helix nemoralis, H. Hortensis, H. Cantiana, etc., Rumina decollata, etc. ${ }^{3}$; cependant les Variabiliana et les Pisana, n'ont pu s'acclimater d'une manière définilive ${ }^{4}$. Cesespeces ne peurent se maintenir el ne tardent pas à s'èteindre en lièrement après cluaque introduction ${ }^{5}$. Ce fait est d'autant plus curieux que la faune malacologique de l'Amérigute du Nord est d'allure bien plus méridionale que notre faune française. Peut-être les Pisania el les Variabiliana, tout en pouvant s'acclimater dans un pays à climat relativement froid, ne samaienl-ils supporter un climat phus chaud, mais oil les hivers sont plus rigoureux.

Les espèces méridionales acclimatées anx environs de Paris recherchent les lieux bien abrités des vents froids du Nord et de l'Est, les Lalus parfititement exposés au midi, où

chroa candidissima qui vivaient aux environs d'Aix-en-Provence, sur des rochcrs cxposés en plein midi, l'une non loin du viaduc de l'Arc, l'autre à la montée d’Avignon.

1 Mabille (J.), 187o. Mist. Malacol. Bassin parisien, p. 99.

2 Locard (A.), 158ı. Marial. Malacol. Bassin Rhône, t. Il, p. 143.

3 Germain (L.), 19o3. El. Moll. Maine-ct-Loire, p. 4o, note 2.

4 Voyez: Forbes, 1880. Brilish Assoc. rep., p. 145 ; Boston Journ. Nat. list., 11I, p. 489. - Binney (IV.), 1878 . The terrest. air breath. Moll... Unit. St. ete..., I (in Bull. of Mus, of Comp. Zool. Gambridge, vol. IV, P. 256 et 257 .

5 Binney (VV.), 1886. A second suppl. of fifth vol... Air breath. Moll. In. St, (in Bull. of Mus of Comp. Zool. Cambridge, vol, Xill, no 2, p. 24). 
ils grimpent, bravant les chauds rayons du soleil, sur les tiges sèches des graminées. Lorsque de telles stalions possèdent une belle végétation et sont, en outre, à proximité de rivières ou d'étangs entretenant une lumidité constante, la colonie prospère et ses individus atleignent une forte taille; si, au contraire, la station ne possède qu'une végétation paurre el chétive, un sol pierreux ou siliceux, la colonie qui, quelquefois sera aussi riche en individus, se composera de coquilles de petile laille, mal venues, parfois d'une remarquable exiguïlé'. Toutes ces espèces étant éminemment calcicoles, prendront leur plus grand développement daus les localités où le calcaire abonde (comme ì Arcueil, Gentilly, etc...), elles disparaîtront là oỉ manque cet élément. C'est aussi qu'elles n’ont pu se développer dans les bois de Clamart, de Saint-Cloud, ete... où, par suite de la présence continuelle du sable, la faune malacologique est si pauve.

Une particularité intéressante est la préférence extrêmement prononcée des Variabiliana pour les chardons. Nous avons déjà, dans la partie descriptive de ce travail, signalé plusieurs fois ce fail, à Clioisy-le-Roi notamment. Les chardons étant répandus à profusion sul les bords de la mer, il $y$ a là une influence marine faible, mais indéniable. Les Onbellifères sèches, les Artemisia, les Foniculmm, et aussi le Centaurea calcitrapa, abondant sur certains talus des fortifications, obticnnent ensuite leur préférence.

1 Sur les planches de elóture des terrains vagues de la rue Earraull, à Paris, les II. fera, II. Avenionensis, etc..., sont remarquables par leur petite taille. 
Généralement les introductions de coquilles méridionales ont lieu, aulour de Paris, vers le milieu du printemps, lorsque le transport des légumes du Midi prend sa plus grande extension. Une telle époque est éminemment favorable à une prompte el parfaite acclimatation : la douceur de la température à celte époque de l'année, la beauté de la végétation qui commence à envahir les localités favorables, tout concourt à fixer les Variabiliana autour de nous; cependant le climat parisien n'est plus, tant s'en faut, celui de la chaude Provence; il est sujet à de brusques refroidissements inconnus du Midi; aussi un tel déplacement rapide a-t-il une influence considérable et arrête-t-il, pour un temps plus ou moins long, le développement des coquilles. L'observation montre que toutes les coquilles subissent alors un arrêt brusque provenaut, sans nul doute, de la différence d'habitat el de température et que, s'apprêtant alors à hiverner, elles se construisent hàtivement un bourrelet apertural. Mais, le Mollusque s'acclimatant peu ì peu sous l'influence de la chaude température estivale, continue jusqu'à l'automne son développement normal interrompu et consolide son ourerture provisoire; il en résulte souvent la formation d'un second bourrelet apertural construit à quelques millimètres de l'ancien. Cependant, il faudrait bien se garder d'altribuer à un tel mécanisme tous les cas de bourrelets multiples que l'on est susceptible de rencontrer. On observe, en effet, chez quelques Helix vivant dans leur milieu normal, la présence de ce double bourrelet; il existe même très fréquemment chez certaines espèces el a pu ùtre pris 
comme un caraclire apertural. Nous avons observé, chez un Ilelix Salentina, Blanc, de Choisy-le-lioi, denx bourelets parfaitement marqués espacés de plus de l centimètre ${ }^{1}$; la mème anomalie a élé signalée chez ın II. Pisana de Bonifacio?. Jusqu'ici ricn ne paraît anormal à l'extériem: mais, lorsque ce phénomène dëjà si curieux s'exagère, il se développe denx onvertures superposées : sur une première ouvertmre normale, une seconde vient s'emboiter et fournir à son tour un péristome complet, un pen moins marqué cependant que celui de la première ouverturc. Celte monstruosité, déjà observée par de Blainville, a été éludiée par Carlo Porro qui la désigne sous le nom de sopra eccilazione di vila ${ }^{3}$. Connue aujourd'hui sous le nom d'hypersécrétion aperturale, cette anomalie a élé signalée chez plusienrs espèces, notamment chez les IIelix aspersa, $I$. lapicida, II. pomatia, II. pyrgia, H. vermiculata, II. nemoralis, 1I. hortensis, II. subaustriaca, Limmea turgida, elc... ${ }^{4}$.

Il est bien évident que les phénomènes précédents ne s'observent quau moment de l'introduction dans la faune locale d'une coqnille déjà d'un certain àge, mais non cncore adulte; chez les colonies acclimatées, les individus se développent plus normalement; les coquilles jeunes commencent a se montrer dès la fin mai el ne progressent d'abord que très lentement. Dès juillet, l'accroissement dans la taille se manifeste pleinement; cependant le péristome n'est pas

1 Hous possédons un individu exlrêmement jeune de J'Il. Pisana qui a Jéjì, à l'intérieur de son ouverture, deux hourrelets trés marqués.

2 Locard (1.), I88ı. Variat. malacol. Bassin Rhône, t. II, p. 197.

3 l’orro (Carlo), 1839 . Studii su talune variasioni offerte da Molluschi nuviatilic lerrestri a conchylia univalve, p. ry.

4 Locard (1.), is81. Tarial malacol. Bassin Rhône, t. II, p. 195-196-Aux exemples signalés dans cet ouvrage, nous ajoulerons ceux observís par 1. Madoulé sur II. nemoralis et $I$. arhustorum aux Moulineaux près Elbeuf. Madoulè (A.), 189 ı. Anom. olıs, chez llélices, in Bull. Soc. Sc. Nat. Elbeuf, so, p. 5 . 
encore formé et e'est à peine si, chez la grande majorité des individus, on observe mn léger épaississement de celle partie de la coquille ${ }^{1}$. Nos Helix construisent done leur ouverture en plein été et, vers la fin septembre, ils sont à peu près adultes presque partout. Ils vivent alors jusqu'au milieu de l'automne mais, très sensibles anx variations de température, ils disparaissent avee les premières gelées. Cependant si, à ces premiers froids, suecèdent des jours plus doux et pluvieux, quelques espèces plus robustes comme les Ilelix pilula, H. fera, H. Mendranoi, se rencontrent à nouvean dans les endroits très abrités, sur les tiges desséchées des Chardons et des Centaurées; mais ces apparitions ne sont qu'éphémères, et une seconde période de froids, même légers, les fait disparaitre. La date la plus extrême où nons ayons relevé de telles colonies est le 27 novembre. Quant aux individus adultes qui ont hiverné, ils n'apparaissent que bien plus lard que les espèees de la faune autochtone : le premier que nous ayons observé en 1903 a élé récolté ì Choisy, le 18 mars: la teripérature s'étail ciéjà, durant plusieurs jours, maintenue is un niveau cxceptionnel pour la saison (21 degrés). Les résultats de l'accouplement des Variabiliana parisiens sont les mêmes que dans les pays d'origine: les individus semblent tont anssi prolifiques, les colonies tout aussi populeuses que celles que nous arons en si souvent oceasion d'observer dans les départements du midi de la France.

1 Sur pres de 1500 Variabiliana reeueillis au milieu de juillet a Choisyle-Roi, près le chemin de fer du P.-I..-M., nous n'avons trouvé que trois coquilles à peu près adultes. 
Lin résumé, ees multiples observations montrent, qu'it part un retard en somme insignifiant dans le développement, une période d'hivernement plus longue, les Variabiliana se sont parfaitement acelimatés dans la banliene parisienne, ils y ont formé des variélés, à la vérité plus robustes, plus rustiques que celles du Midi, mais supportant parfaitement désormais le elimat si inégal clu Bassin de Paris. Ces rariétés ont subi certaines modifications que l'on observe tonjours chez les types meridionaux passant dans un pays plus septentrional el qui penvent se résumer de la manière suivante :

$1^{\circ}$ Diminution sensible dans la taille: les variétés minor. se rencontrent volontiers, ainsi que nous l'avons signalé ehez un certain nombre d'espèces : Ilelix A venionensis, H. Mendranopsis, H. acompliella, H. Jera, H. Cyzicensis, I1. Mendranoi, H. limbrifera, H. leviculina, elc.

$2^{0}$ Les formes déprimées ou subdéprimées tendent à devenir subglobuleuses ou même globuleuses (Ex. H. ambielina, II. fera, H. nemausensis, etc), landis que les espèces très liautes et coniques, d'ailleurs rares partout, voient leur galbe s'abaisser el deviennent globuleuses élevées ou moins franehement coniques ${ }^{1}$. Tel est le eas des IIelix Tabarkana, H. scicyea, H. melantozona, II. lineata, etc...

1 C'est à des échantillons de l'II. limarella et de l'H. limbifera possédant un galbe surbaissé, mais dont les tours sont devenus trop convexes et la suture trop profonde (par suite d'une exagération de la tendance indiquée préé́demment) que l'un de nous a donné le nom d'Ilelix Leontinei, Germain, (1898. Deseript. esp. nouv. Helix, in Bull. Soc. Am. Sc. Nat. Rouen, XXXIV, p. 197). 
Nous avons déjà observé, en Maine-et-Loire, un phénomène de même ordre: les Helix lineata ct H. scicyca, qui vivent sur les rocher's dévoniens de la station méridionale de Beaulieu sont, tout en restant très typiques, un peu moins élevés que sur le littoral méditerranéen; mais le climal de Beaulieu étant beancoup plus chand, cette tendance, à peine sensible, est bien moins marquée que ehez les coquilles parisiennes.

3० Diminution dans l'épaisseur du test; en réalité, e'est cette particularité qui est la moins constante et la moins sensible; cependant, nous l’avons constatée généralement chez les Helix acomptiella, II. scicyca, II. lineata. Cetle loi est eependant très normale, on l'observe presque toujours lorsque l'on compare, par exemple, les formes du Midi de la France avec les formes similaires dı Maroc, de l'Algérie et de la Tunisie; les premières ont le test normalement plus mince que les autres : ayant moins à redouter l'influcnce des chauds rayons solaires qui tend tonjours à leur faire perdre l'humidité qui leur est si nécessaire, elles n'ont pas besoin, dans le Nord, d'édifier une demeure à parois aussi épaisses.

$4^{\circ}$ Allénuation très sensible dans la coloration. Chez les coquilles unicolores la teinte est plus pâle et ne possède pas ce brillant que l'on retrouve chez toutes les coquilles méridionales et aussi, quoique alténuée déjà, chez les Mollusques de l'Anjou. Chez les espèces olnées de bandes, ees dernières, si elles ne s'oblitèrent pas complètement, sont toujours plus étroites, moins ehaudement colorées el aussi moins transparentes ${ }^{1}$. Sourent elles s'effacent entièrement : tel est le cas

1 En Anjou, les formes fascices comme $I$. Cy sicensis, $/$. scicyca, elc..., ont un fond llane brillant, crétacé, orné de bandes très chaudement colorées comme dans les échantillons de la Provence [Germain, 1893 . Et. Moll. Maine-et-Loire, p. 120-128]. 
de la colonie de II. Pisania, de Charenton. Les individus qui, au début, étaient fasciés ont, au bout de quelques générations, complètement perdu leurs bandes ornementales : lous ont aujourd'hui un test blane un peu jaunâtre, légèrement brillant, analogue à celui des Ilelix Carpiensis, Lel. et Bourg ${ }^{1}$, et II. Bertini, Bourg'², du littoral méditerranéen. C'est à peine si, eliez quelques rares échantillons, on peut distinguer quelques traces de faseies très obsolètes aux environs immédiats de l'ouverture ${ }^{3}$. Autour de Lyon, ou l' $H$. I'isana a été également introduit, les bandes s'atlénuent plus ou moins complètement, sans disparaître cependant ${ }^{4}$; mais la coloration rosée de l'ouverture ne persiste pas $^{5}$, ce qui n’a pas lieu, généralement du moins, chez la coquille de Charenton ${ }^{6}$. Du reste, toutes les coquilles, soit terrestres soit marines, qui vivent dans les pays chauds sont toujours plus chaudement colorées el plus richement décorées que celles des pays tempérés. La faune abyssale de tous les pays conserve seule ces tons pàles, comme chlorotiques, propres à la plupart des espèces de la faune septentrionale ${ }^{7}$.

Tandis que la très grande majorité des espèces que nous avons eu à signaler se sont parfaitement acelimatées, d'autres, en petit nombre, ne semblent qu'aberrantes, ou du moins ont eu beaucoup plus à souffrir de leur déplacement. Tel est le cas des IIelix à galbe conique-élevé en dessus du groupe

1 Letourneux et Bourguignat, 1887. Prodr. Malacol. Tunisie, 1). So et 86.

2 Bourguignat, in Locard, 1882 . Prodr., p. 103 et 329 .

${ }^{3}$ On rencontre très rarement à Charenton quelques individus fasciés de I'II. I'isana, ils sont évidemment d'introduction récente. Tel est le cas du seul ichantillon que nous ayons recueilli dans cette station en plusieurs années.

4 Locard (A.), 1881. Varial. malacol. Bassin Rhöe, t. I, p. 148.

5 Locard (A.), 1881. Varial. malacol. Bassin Ihhöne, t. II, p. f04.

- A Charenton, les II. Pisana ont souvent l'intérieur de l'ouverture coloré en lose assez vif.

־Locard (A.), 18y5. Mollusques testacés du Travailleur el du Talisman, t. $11, \mathrm{P} \cdot 466$. 
de l'Helix lineata. Presque toules ces formes haules, rares parlout el plus ou moins localisées ${ }^{1}$, ne s'éloignent que difficilement des régions sounises à l'influence marine, aussi leur acclimalement ne réussit-il pas loujours. Un remarquable exemple nous est fourni par une espèce très répandue d'un genre voisin : le Cochlicola barbara, qui remonte fort haut sur les côtes, puisqu'on en constate la présence sur les rives de l'Angleterre et du Danemark. Cependant cette coquille, signalée maintes fois à l'intérieur, n'a pu se maintenir lá où l'influence marine ne se faisait plus sentir. Elle a entièrement disparu des environs de Lyon ${ }^{2}$ et n'a pu davantage prospérer dans la plaine d'Ardenay ${ }^{3}$ (Sarthe). De temps à autre, on peut recueillir le C. barbara anx environs de Paris, le long des berges de la Seine, où il est apporté par les nombreux bateaux marchands qui font le service du Havre. C'est ainsi que Bourgnignat l'a récolté sur le quai de Javel, dans Paris même ${ }^{4}$ et que M. Mabille nous a dit l'avoir recueilli, en magnifiques échantillons, dans les îles de la Seine, un peu au-dessous du viaduc d'Auteuil. Le point le plus éloigné où nous connaissions cette espèce est jusqu'ici Champtocé, dans le déparlement de Maine-et-Loire, oì elle est abondante et où Millet l'a vait signalée dès $1813^{5}$.

Les Ilelix du groupe du lineata des environs de Paris se ressentent évidemment de la grande distance de la mer et leur galbe esl beancoup plus éloigné du type que celui des

1 A I'cxception toutefois des Ilelix lineala, II. melantozona, II. urnina, II. foelatina, qui sont des formes communes dans certaines régions méridionales.

${ }^{2}$ Locard (A.), 1877. Malacol. lyonnaise, p. 49. - Locard (A.), 1878 . Migr. Malacol. Lyon, p. 8. - Locard (A.), i $\$ 82$. Contiib., IV, p. 14.

3 Ituard, in Morin, 1883. In Bull. Soc. Agric. Sc. Arts Sarlhe, XXIx, p. fo2. - Morin, 1894 . Faun. malacol. Sarthe, p. 36.

4 Locard (1.), 1882. Contrib., IV, p. 14.

5 Millet (P.-A.), 1813 . Moll. Maine-et-Loire, p. 41, n 4. - Germain (L.), 1903. Etude Mollusques Maine-et-Loire, p. 129. 
autres espèces introduites. Quelques espèces, comme les $H$. siscyca et $H$. Tarbakiana, n'ont pas semblé pouvoir s'aeclimater et, malgré nos recherches, nous n'avons pu les retrouver au square Alboni, seule localité où nous les ayons récoltés. Mais toutes les autres Hélices que nous avons signalées sont bien définitivemeut acclimatées et, selon toute vraisemblance, ne disparaîtront plus de nos environs. Quelques-unes, très rustiques, étendent constamment leur aire de dispersion el tendent à envahir loute la région; elles se multiplient en telle abondance, qu'elles sont déjà une cause d'ennui pour les cultivateurs et les jardiniers qui ne parviennent que fort difficilement à s'en défaire.

Parmi les lerophila, les espèces voisines des 11 . variabilis, H. Pisana ${ }^{1}$, etc... sont, pour la plupart, d'origine récente et ne remontent guère au delà du Pleistocène ${ }^{2}$, leur's formes ancestrales ne nous sont pas encore bien connues. En tous les cas, le centre d'apparition de ees eoquilles parail être le Bassin Méditerranéen. Très abondantes sur toutes les côtes françaises de la Méditerranée, mais surtout dans la région provençale, elles n'ont pas traversé les Alpes qui semblent, pour ces Hélices, une barrière infranchissable; la plupart des formes italiennes appartiennent en effet, ì des groupes différents de ceux de la faune française. A ees Mollusques il faut un climat relativement chaud et mari-

1 IIelix depicla [Grataloup, 1839. Soc. Lin. Bordeaux, t. XI, p. 399, pl. I, fig. 12].-Binney (W.) [in Bull. of . Mus.Comp. Zool. Cambridge, IV, 1878, p. 256] considère celte coquille comme une forme naine et non ombiliquée de l'H. Pisana.

'Locard (A.), 1882. Var. malacol. Bassin Rhône. 1. 1I, p. 165. 
lime: les hivers trop vigoureux leur sont extêmement nuisibles et fonl souvent disparaître des colonies déjà prospères ; l'influence marine est un facteur actif el souvent indispensable de prospérité: ces conditions climatériques suffisent pour expliquer la dispersion des Variabiliana en France.

A côté des déplacements brusques étudiés précédemment, nous allons constater maintenant de véritables migrations. Ces migrations se sont faites lentement et dans deux directions :

$1^{\circ}$ Quelques espèces, à la vérité peu nombreuses, ont remonté la vallée du Rhône, mais n'ont guère dépassé le département de Vaucluse. quoique quelques I'ariabiliana aient étć signalés jusque dans la Drôme ${ }^{1}$ (II. Pisana, II. Cyzicensis, II. Xalonica); telles sont, par exemple: Ilelix stiparm, Rossm., H. Vardonensis, Loc., II. Pisanorum, Bgt., I1. terraria, Loc., I1. Salentina, Blane. 11. Montgiscardiana, Fag., H .Canovasiana, Serv, efc.:

$2^{\circ}$ D'autres, en beancoup plus grand nombre, suivant les lignes ou mieux les zones isothermes, ont pénétré dans la vallée de la Garonne el, remontant le littoral océanique. traversant les estuaires de la Gironde el de la Loire ont, toujours sous l'influence d'un elimat dont les rigueurs sont alténuées par l'action bienfaisante du Gulf-Stream, franchi le cap Finistère el essaimé sur le littoral de la Nanche. Parmi ces formes méridionales qui ont ainsi émigré jusque dans la Manche, nous signalerons: H. sphxrita, Hartm. (Morbihan); Il. arenarum. Brgt. (Morbihan); II. Auglustiniania, Brgt. (Allantique el Manche); H. limbifera, Loc. (Allantique el Manche); H. calculina, Loc. (Vendée. Calvados); H. acomptiella, Loc. (Finistère); II. Evenosi. Brgt. (Atlantique); II. ademata, Brgl. (Atlantique); $H$.

\footnotetext{
- Chatenier, 1888. Cial. moll. ter. /luv. Drome, 1'. 20.
} 
Mendranopsis, Loc. (Atlantique, Manche); H. Nemansensis, Brgl. (Atlantique, Manche); H. Avenionensis, Brgt. (Atlan(ique); II. Guideloni, Brgt. (Atlantique); H. fera, Let. Brgt. (Atlantique, Manche); H. Grannonensis, Brgt. (Atlantique, Manche); $I$. variabilis, Drap. (Allantique, Manche); $H$. Nalonica, Serv. (Atlantique, Manche); H. alluvionum, Serv, Atlantique, Manche): 11 . Cysicensis, Gall. Atlantique, Manche); I1. Mendranoi, Serv. (Allantique, Manche); 11. Mendoza, Serv. (Atlantique, Manche); H. papalis, Loc. (Atlantique, Manche); $H$. mucinica, Brgt. (Atlantique. Manclie); II. scicyca, Brgi. (Atlantique, Manche); II. Sylvie, Serv. (Allantique, Manche); H. pilula, Loc. Allantique, Manche); II. Ogiaca, Serv. (Atlantique, Manche); II. migrata, Loc. (Atlantique, Manche): H. lineata. Olivi (Atlantique, Manche); H. melantozona. Caf. (Atlantique); If. urnina, Loc. (Allantique); H. fodata, Hagenm. (Atlan (ique); II. foedatina, Loc. (Atlantique, Manclie); 11. Tabacliana, Let. Brgt. (Allantique); etc...

Si maintenant nous cherchons à établir un parallèle entre notre nonvelle faumle adventice parisienne avec la même faunule normale olsservée dans d'autres pays, nous constaterons que, sur les 47 espèces que nous avons relevées jusqu'à présenl, nous en retrouvons 43 sur les côtes de Provence, 12 en Tunisie, 16 en Algérie, 9 en Italie, 13 en Espagne el i 8 en Portugal. D’autre part, sur les 14 espèce: acclimatées dans les mêmes conditions aux environs de Lyon, 1 I sont communes à ces demx régions.

En outre, il est à remarquer que les Tariabiliana sont de moins en moins nombreux à mesure que l'on remonte vers le Nord et qu'un nombre fort restreint de formes ont pu traverser l'estuaire de la Seine. Le Morbiluan et le Finistère semblent, à première vue, plus riches que les départements plus méridionanx de la Loire-Inférieurc et de la 
Vendée; mais cette exception n'est qu'apparente et tient au climat exceptionnellement tempéré de la Bretagne qui bénéficie de l'épanouissement de la branche supérieure du Gulf-Stream.

Observons enfin que certains départements, qui ne sont pas littoraux, possèdent néanmoins une faune assez riche en Variabiliana. Tels sont le Maine-et-Loire et l'Indre-et-Loire. Cette apparente exception tient à la présence de la Loire, les coquilles introduites ayant tendance à remonter le cours des grands fleuves. Il y a lá un phénomène du mème ordre que celui dont nous avons parlé à propos du Rhône, mais moins marqué, par suite de la latitude plus élevée. Cette tendance s'atténue encore sur la Seine où les Variabiliana ne sortent pas du département de la Seine-Inférieure.

Il existe une parfaite similitude entre les phénomènes migratoires propres anx Mollusques et ceux propres aux végétaux. Partout où l'on a signalé la présence de coquilles méridionales, à Lyon ${ }^{1}$, dans les départements de Maine-etLoire $^{2}$, de la Loire-Inférieure ${ }^{3}$, du Calvados ${ }^{4}$, de la SeineInférieure ${ }^{5}$, etc..., on a également constaté l'existence de plantes méridionales. Comme l'écrivait l'un de nous dès I $882^{6}$, il y a là une grande loi de corrélation entre le monde animal et le monde végétal que nous laissons à d'autres, plus expérimentés que nous, le soin de confirmer par de nouveaux exemples.

1 Saint-Lager $\left(D^{r}\right), 1_{7}$. Note sur intr. quelqués plantes mérid. à Lyon et dans ses environs, in Ann. Soc. Bot. Lyon, t. I.

2 Boreau (.1.), 1859 . Calalogue plantes vascul. Maine-et-Loire. - Boreau (A.), 1859 à 1866 . Princip. herl. Maine-et-Loire, 8 br. in-8, Angers.

${ }^{3}$ Llyod"(J.), Flore de l'Ouest de la France, $4^{\mathrm{C}}$ édit.

* Corbière, Nouv. flore Normandie.

5 Toussaint et Hoschedé, 1897. Flore Vernon, Toche-Guyon, elc., in Bull. Sm. Sc. Nat. Rouen, XXXIII, p. 103.

${ }^{6}$ Locard (A.), 1882. Contrib., IV, p. 84. 
Il semblerait que de tels faits soient contraires à toutes les notions généralement admises sur la distribution géographique des Mollusques. Il n'en est rien, cependant, et nous venons de voir que les migrations des espèces ne se font pas au hasard, mais selon des règles assez précises pour que nous ayons pu les formuler. Nous les résumerons de la manière suivante :

$r^{\circ}$ Les espèces méridionales ne peuvent s'acelimater que dans un pays possédant des conditions climatériques similaires à celles du pays d’origine; dans les régions oì la moyenne annuelle est plus faible, l'aeclimatement définitif n'a lien que si les hivers ne sont pas rigonreux;

$2^{\circ}$ Lorsqu'une espèce méridionale, brusquement transportée dans une région plus septentrionale s'est acelimatée, elle donne naissance à des variétés plus robustes, adaptées au nouveau milieu;

$3^{\circ}$ Les espèces méridionales, en dehors des importations brusques du fait volontaire ou involontaire de l'homme, peuvent émigrer en se déplaçant lentement, d'elles-mêmes, de proche en proche; elles suivent les lignes ou zones isothermes;

$4^{\circ}$ Ces déplacements n'intéressent qu'une bande littorale de faible épaisseur, les points les plus éloignés du rivage où l'on constate ces espèces restant encore soumis à l'influence maritime ;

50 L'aire de ces déplacements n'est pas illimitée : il existe une zone-limite que les Mollusques ne peuvent franchir et dont le eritérium est fourni par la température au-dessous de laquelle les espèces ne peuvent s'acelimater. Pour les IIélices méditerranćennes qui vivent sur les côtes de l'Atlantique el de la Manche, eette zone-limite est comprise entre l'embouchure de la Seine et le Pas-de-Calais ;

6. Les Mollusques migrateurs, lorsqu'ils font élection 
d'un domicile nouveau, choisissent toujours de préférence les stations les mieux exposées, celles qui sont le plus conformes à leur modus vicendi normal, et, en particulier, celles qui sont à l'abri des vents ;

$7^{\circ}$ En général, les colonies nouvelles, même lorsqu'elles sont très populeuses, sont très localisées et ne se dispersent que très lentement;

$8^{\circ}$ Les espèces méridionales ont une tendance à remonter les vallées des grands fleuves; elles remontent d'autant plus haut quelles sont plus près de leur centre de dispersion. Il en est de même des espèces subeosmopolites;

$9^{\circ}$ La direction des fleuves n'a aucune influence sur la migration des espèces. 

$65^{694}$ 
